\title{
SONDIERUNGS- UND EVALUATIONSSTUDIE „YOUNG SCIENCE - ZENTRUM FÜR DIE ZUSAMMENARBEIT VON WISSENSCHAFT UND SCHULE"
}

im Auftrag des Bundesministeriums für Wissenschaft, Forschung und Wirtschaft

(GZ BMWFW-37.679/0001-WF/V/4/2014)

Caroline Manahl

Susanne Dobner

Teresa Holocher-Ertl

Barbara Kieslinger 


\section{Inhaltsverzeichnis}

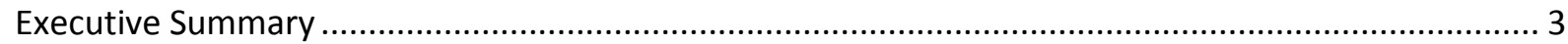

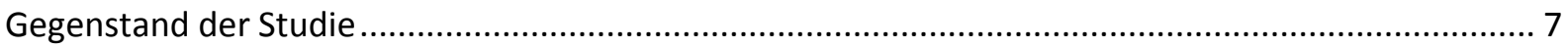

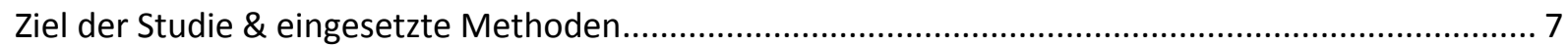

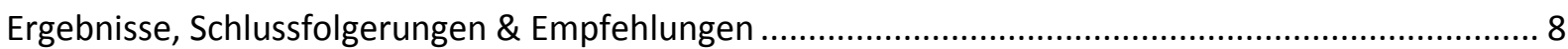

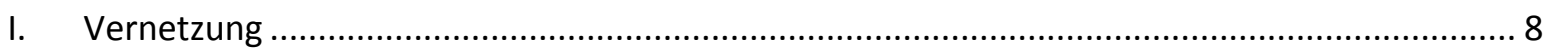

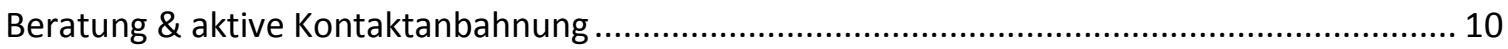

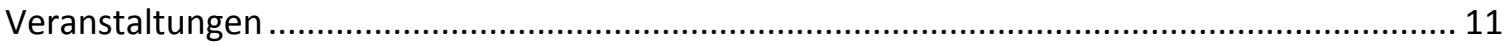

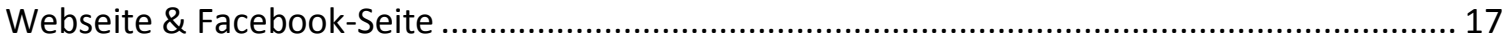

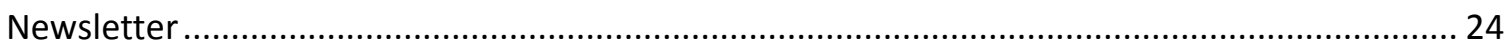

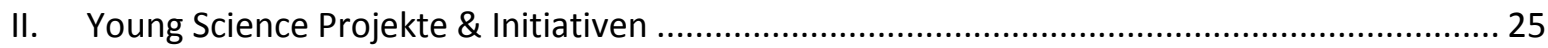

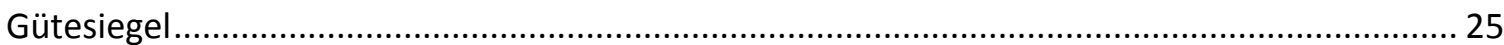

Themenplattform für vorwissenschaftliche Arbeiten ................................................................. 29

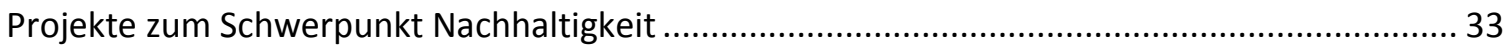

Entwicklungspotentiale im Bereich Citizen Science …....................................................................... 35

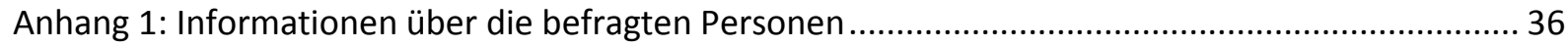

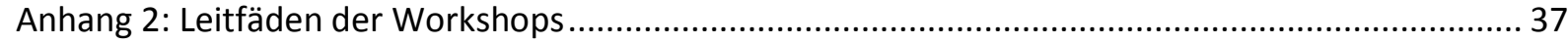

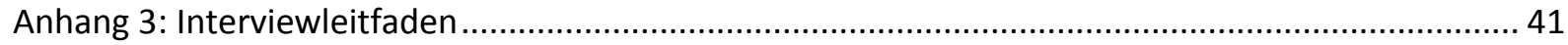

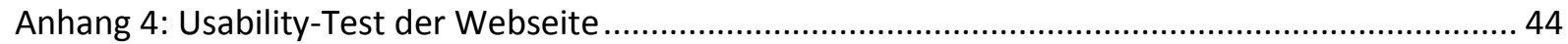




\section{Executive Summary}

Young Science wurde 2011 als Projekt mit dem Ziel der Förderung der Zusammenarbeit von Schulen und Wissenschaft im OeAD eingerichtet. Primäre Zielgruppen des Projekts sind LehrerInnen, BildungsberaterInnen, SchülerInnen, ForscherInnen und Personen, die an der Schnittstelle zwischen Schule und Wissenschaft arbeiten. Seit 2011 hat sich Young Science von einer Informations- und Servicestelle zu einer Plattform weiter entwickelt, die zunehmend eigene Projekte und Initiativen umsetzt. Diese Evaluierungs- und Sondierungsstudie setzte daher zwei Schwerpunkte - einerseits Vernetzungsaktivitäten und andererseits Young Science Projekte. Qualitative und quantitative Methoden der Datensammlung und Auswertung kamen dabei zur Anwendung.

Die quantitativen Auswertungen von Webstatistiken und Veranstaltungsdaten gaben Aufschluss über die Entwicklung der Anzahl der Kontakte in die Zielgruppen und deren Bewertung von Veranstaltungen. Zusätzlich wurden im Dezember 2014 zwölf VertreterInnen der Zielgruppen und MitarbeiterInnen von Young Science mittels Workshops und Interviews in die Evaluierung einbezogen, um den Nutzen und die Motivatoren der Teilnahme an Young Science-Aktivitäten besser zu verstehen bzw. auch Barrieren in der gemeinsamen Arbeit zu identifizieren.

Das qualitative Feedback aller TeilnehmerInnen aus Workshops und Interviews, sowie zunehmende BesucherInnenzahlen bei off- und online Aktivitäten unterstreichen die hohe Relevanz des Young Science Zentrums. Der Bedarf eines Mittlers zwischen Schulen und Wissenschaft wurde klar geäußert. Die Rahmenbedingungen für diese Zusammenarbeit sind dabei sowohl auf der Schul- als auch auf der Wissenschaftsseite noch schwierig und können durch die Aktivitäten des Young Science Zentrums positiv unterstützt werden. Auf beiden Seiten sind für die Forschungs-BildungsKooperation kaum Ressourcen, sowohl im zeitlichen als auch materiellen Sinne, vorgesehen. So stellen gemeinsame Forschungsprojekte für alle Involvierten (LehrerInnen, ForscherInnen, SchülerInnen) oftmals einen Zusatzaufwand da, der immer wieder in der eigenen Freizeit abgewickelt wird, und wenige monetäre bzw. nicht-monetäre Anreize bietet. Motivierte LehrererInnen und WissenschafterInnen sind der Schlüssel zum Erfolg der Zusammenarbeit. Oft ist diese Motivation allerdings intrinsischer Natur (z.B. das große Interesse am Forschungsthema und das Anliegen, SchülerInnen für die Forschung zu interessieren). LehrerInnen berichten einerseits von unflexiblen Lehrplänen, hohem administrativen Aufwand bei der Abrechnung von z.B. Reisekosten, und heben die positiven Erfahrungen aus den Projekten hervor. Dazu zählen: die Möglichkeit zur Beschäftigung mit aktuellen Themen, wie z.B. Nachhaltigkeit, der Einblick der SchülerInnen in die Wissenschaft, geringere Berührungsängste und größeres Interesse der SchülerInnen durch die kontinuierliche Präsenz von ForscherInnen, sowie die Erweiterung des Wissens sowohl bei Schülerlnnen als auch bei LehrerInnen. Betont wird auch, dass die Teilnahme an Forschungsprojekten oder beim Gütesiegel bei der Bewerbung der Schule hilft.

ForscherInnen sind neben der intrinsischen Motivation vor allem an Drittmitteln zur Finanzierung ihrer Projektvorhaben interessiert. Zeit und Ressourcen sind für die Zusammenarbeit mit Schulen darüber hinaus kaum vorhanden. Zur Förderung der aktiven Einbindung von SchülerInnen in Forschungsarbeiten, wäre die Berücksichtigung des "Social Impacts“ in wissenschaftlichen Bewertungsschemen, sowie ein Erfahrungsaustausch, der den Nutzen dieser Einbeziehung auch für die wissenschaftliche Arbeit hervorhebt, von großer Bedeutung.

Aufgrund dieser Rahmenbedingungen wird eine hohe Serviceorientiertheit von Young Science verlangt. Die Herausforderung liegt darin, mit möglichst kurzen und prägnanten Informationen das 
Interesse der Zielgruppen zu schüren, Kontakte und diverse Einstiegsmöglichkeiten (z.B. relevante Veranstaltungen für die Zielgruppen) zu ermöglichen. Einerseits sollen so neue Teilnehmerlnnen gewonnen und bereits Interessierte immer wieder an das Thema erinnert werden. Andererseits benötigt es für konkrete Fragen und Vorhaben eine hohe Flexibilität und Verfügbarkeit seitens der Young Science- MitarbeiterInnen, um Detailinformationen zur Verfügung zu stellen.

Unter dem Motto „Der Prophet muss zum Berg kommen“, muss Young Science dort anzutreffen sein, wo die Zielgruppe ist, und einen klaren Nutzen und Verfügbarkeit zeigen, für jene, die sich mehr interessieren. Young Science ging in dieser Richtung bereits sehr erfolgreich erste Wege. So wurde von allen Befragten die hohe Verfügbarkeit der MitarbeiterInnen via E-Mail, Telefon und persönliche Gespräche betont.

Beiträge, die im Zuge anderer Veranstaltungen für bestimmte Zielgruppe (wie Fortbildungen von BildungsberaterInnen und Tagungen von DirektorInnen) von Young Science gemacht werden, werden ebenso sehr geschätzt. Diese Inputs tragen bei Personen, die Young Science schon kennen, dazu bei, Services in Erinnerung zu rufen oder neue Services vorzustellen. Bei Personen, die Young Science noch nicht kennen, haben sie den großen Vorteil, dass die Personen, die hinter Young Science stehen, kennen gelernt werden. Und wie Workshops und Interviews zeigen, ist gerade bei der Vernetzung von Personen mit geringen zeitlichen Ressourcen die persönliche Ebene sehr wichtig. Daher empfiehlt es sich, solche Informationsangebote in Zukunft weiter auszubauen.

Auch die Idee, BotschafterInnen an Schulen einzusetzen, um neue AkteurInnen zu erreichen, ist sehr vielversprechend. Als weitere Orte zur Zielgruppenerschließung bieten sich Museen, Messen für LehrerInnen, online Ausbildungsplattformen für LehrerInnen, Plattformen die online Lernressourcen aus der Forschung zur Verfügung stellen, etc. an.

In Bezug auf Veranstaltungen zeigen die Analyseergebnisse zudem, dass diese vor allem dann erfolgreich sind, wenn sie einen klaren praktischen Nutzen für die TeilnehmerInnen haben. Daher empfehlen sich kleinere Formate mit einem konkreten Thema und einer spezifischen Zielgruppe (wie beispielsweise das Vernetzungstreffen von Kinderuniversitäten).

Die quantitativen Daten zu den Veranstaltungen zeigen ein sehr positives Bild - sowohl in Bezug auf die Entwicklung der TeilnehmerInnenzahlen als auch in Bezug auf die Zufriedenheit der TeilnehmerInnen. Auch die stärkere Einbindung von SchülerInnen selbst (im Zuge innovativer Formate wie bspw. dem Science Slam), wird von Lehrpersonen positiv aufgenommen, da sie SchülerInnen neue Perspektiven eröffnen und ihr Engagement, das häufig in der Freizeit stattfindet, anerkennen.

Auch die quantitativen Daten der Online-Angebote entwickeln sich mit zunehmenden BesucherInnenzahlen und einer längeren durchschnittlichen Verweildauer positiv. Hier gilt es einerseits, VertreterInnen der Zielgruppe an möglichst vielen Punkten zu erreichen und durch prägnante, kurze Informationen in die Tiefe zu führen. Andererseits sollen jene, die Detailinformationen suchen, mit klaren Anleitungen, Erfahrungsberichten und vor allem Kontaktmöglichkeiten unterstützt werden.

Die Webseite als wesentliches Einstiegsportal zu Young Science bietet den unterschiedlichen Zielgruppen strukturiert Informationen an. Im Usability-Test wurden einige Aspekte deutlich, die in Zukunft noch verbessert werden könnten (z.B. Übersichtlichkeit der Startseite und im Bereich Kontaktpersonen, konsequente Verlinkung von Inhalten mit allen Zielgruppen-Rubriken, für die die Informationen relevant sind). 
Der Newsletter verzeichnet jährliche Neuanmeldungen und ist ein weiteres Instrument, um Informationen an die Zielgruppen zu streuen. Kürze und Prägnanz der Information spielen hier eine wesentliche Rolle und sollten beachtet werden. Konkreter Informationsbedarf zu bestimmten Themen könnte über seltener erscheinende Themen-Newsletter (wie z.B. zum Thema VwA) abgedeckt werden und somit mögliche Synergieeffekte schaffen.

Anstrengungen, sich über Facebook stärker mit SchülerInnen zu vernetzen, waren zwar bisher wenig erfolgreich, sollten aber weiter verfolgt werden. Regelmäßiges posten von News zu Schulprojekten oder zu neuen Themen in der Themenplattform, sowie die aktive Einbindung von Schülerlnnen durch Wettbewerbe könnten sich eigenen, um SchülerInnen auf Young Science aufmerksam zu machen.

In der Themenplattform für vorwissenschaftliche Arbeiten wird von Lehrpersonen großes Potential gesehen - insbesondere wenn es darum geht, SchülerInnen bei der Themenfindung zu unterstützen. Konkrete Erfahrungen mit der Plattform wird es vermutlich erst im Sommersemester 2015 geben. Bei der Entwicklung der Plattform wurden allerdings Schwierigkeiten sichtbar, die für die Arbeit an Schnittstellen typisch sind. Durch Aktivitäten verschiedener Organisationen kam es zu Abstimmungsproblemen und Mehrfachanfragen an ForscherInnen, die bei Personen mit knappen Zeitressourcen für Unmut sorgen. Hier erscheint nicht nur eine sehr enge Zusammenarbeit mit anderen Initiativen, die sich mit der VwA beschäftigen, sondern auch eine klare Abgrenzung der Zuständigkeiten und Betätigungsfelder sinnvoll. Durch den hohen Bedarf an Information in Zusammenhang mit der VwA, der nicht nur an Universitäten sondern auch an Schulen besteht, können Erwartungen an Young Science entstehen, die nicht erfüllt werden können (z.B. Hilfestellung für Lehrpersonen in Zusammenhang mit sozialwissenschaftlicher Methodik). Young Science könnte hier in Zukunft eine wichtige Rolle bei der Kommunikation von strukturellen und gesetzlichen Rahmenbedingungen, in die die VwAs eingebettet sind, zukommen, um alle Beteiligten ausreichend zu informieren und Ablehnungen zu vermeiden.

Das Young Science-Gütesiegel zeichnet seit 2012 Schulen mit einem herausragenden Engagement in der Forschungs-Bildungs-Kooperation aus. Schulen, die mit dem Gütesiegel ausgezeichnet wurden, sehen darin eine Stärkung des Schulprofils und die Möglichkeit von neuen, auf Gütesiegel-Schulen abgestimmte Projekte zu profitieren. Allerdings zeigt sich eine Konzentration von prämierten Schulen in Ostösterreich bzw. in Städten, die mit der dortigen Konzentration von Forschungseinrichtungen und dem - im Vergleich zum ländlichen Raum - verstärkten Wettbewerb um SchülerInnen in Zusammenhang stehen dürfte. Bei den Schulen, die mit dem Gütesiegel ausgezeichnet wurden, handelt es sich fast ausschließlich um Schulen, die zu Abschlüssen mit Studienberechtigung (AHS/BHS) führen. Eine zukünftige Herausforderung dürfte folglich darin bestehen, die ForschungsBildungs-Kooperation für ländliche Regionen und andere Schultypen attraktiver zu gestalten. Mögliche Entwicklungspotentiale zur Ansprache der ländlichen Regionen und anderer Schultypen könnten durch die Forcierung von Citizen Science Projekten entstehen. Citizen Science hat große Potentiale für ForscherInnen und SchülerInnen ${ }^{1}$ und bietet einen niederschwelligen Zugang zu Forschung. Gerade in ländlichen Gebieten könnten hier gemeinsame Themen gefunden werden, von denen alle Beteiligten profitieren.

\footnotetext{
${ }^{1}$ siehe Holocher-Ertl, T., Kieslinger, B. (2014): Citizen Science: Definitionen, Ausprägungsformen und Aktionsmodule. Arbeitspapier BMWFW.
} 
Institutionen, die sich bisher schwer in das Young Science-Netzwerk einbinden ließen, sind Pädagogische Hochschulen. Gerade diese bieten aber großes Potential für Young Science, um sich stärker Schultypen wie Neuen Mittelschulen oder Volksschulen zu öffnen. In diesem Zusammenhang könnte verstärkt die Unterstützung des BMBF gesucht werden, um Young Science-Kontaktpersonen zu etablieren.

Zusammenfassend lässt sich eine sehr positive Entwicklung von Young Science feststellen, dessen Potentiale zur Vernetzung von Schule und Wissenschaft noch nicht ausgeschöpft sind. Mit neu entstehenden Initiativen, wie Young Citizen Science, eröffnen sich innovative Möglichkeiten für die Forschungs-Bildungs-Kooperation. 


\section{Gegenstand der Studie}

Inhalt dieser Evaluierungs- und Sondierungsstudie des Zentrum für Soziale Innovation (ZSI), welche vom Bundesministerium für Wissenschaft, Forschung und Wirtschaft (BMWFW) in Auftrag gegeben wurde, ist Young Science - das Zentrum für die Zusammenarbeit von Wissenschaft und Schule. Young Science wurde 2011 als Projekt mit vierjähriger Laufzeit von der österreichischen Agentur für internationale Mobilität und Kooperation in Bildung, Wissenschaft und Forschung (OeAD) eingerichtet.

Ziel von Young Science ist es, durch die Errichtung einer Netzwerk- und Serviceplattform Synergieeffekte bestehender und zukünftiger Initiativen des BMWFW an der Schnittstelle zwischen Schule und Forschung besser zu nutzen, die Kooperation zwischen sekundären und tertiären Bildungseinrichtungen zu fördern und Schnittstellen zum Bundesministerium für Bildung und Frauen sowie anderen Ressorts zu öffnen. Als primäre Zielgruppe von Young Science wurden kooperationsinteressierte Forschungs- und Bildungseinrichtungen definiert ${ }^{2}$, welche mit folgenden Aktivitäten erreicht werden sollten:

- Vernetzungsaktivitäten: Veranstaltungen, Beratung und aktive Kontaktanbahnung, Webseite, Öffentlichkeitsarbeit

- Young Science Gütesiegel

Im Verlauf der letzten vier Jahre wurde Young Science zudem vom BMWFW mit der Umsetzung weiterer Projekte beauftragt:

- Projekte zum Thema Nachhaltigkeit (,Rio +20 - Wissenschaftler/innen und Jugendliche ziehen Bilanz", „Gemeinsam für nachhaltige Entwicklung - The Future We Want")

- Themenplattform für vorwissenschaftliche Arbeiten und Diplomarbeiten

Zudem wurde Young Science mit der Koordination des Projekts „MINI MED Junior“ betraut. Ab Ende 2014 liegt ein weiterer Schwerpunkt von Young Science im Bereich Citizen Science.

\section{Ziel der Studie \& eingesetzte Methoden}

Ziel dieser Studie ist es, die bisherigen Aktivitäten von Young Science zu evaluieren, sowie zukünftige Entwicklungspotentiale auszuloten. Dazu wurden qualitative und quantitative Methoden eingesetzt. Die qualitativen Methoden dienten dazu, Personen, die in den vergangenen Jahren in engem Kontakt mit Young Science standen, sowie die MitarbeiterInnen des Zentrums in die Evaluierung einzubeziehen. ${ }^{3}$ Durchgeführt wurden:

- Workshops (ein Workshop mit vier StakeholderInnen an Schulen und Universitäten, sowie ein Workshop mit drei MitarbeiterInnen von Young Science) ${ }^{4}$

- Leitfadengestützte Interviews (mit fünf StakeholderInnen an Schulen und Universitäten) ${ }^{5}$

Im Zuge der quantitativen Analyse werteten die Autorinnen dieser Studie folgende von Young Science zur Verfügung gestellte Daten aus:

- Veranstaltungsdaten (inkl. Feedback der TeilnehmerInnen)

- Statistiken der Webseite, des Newsletters sowie der Facebook-Seite

- Daten zum Gütesiegel

\footnotetext{
${ }^{2}$ Ziele und Zielgruppen laut Angebot des OeAD und Arbeitsprogramm 2012 von Young Science

${ }^{3}$ Eine Liste der WorkshopteilnehmerInnen und InterviewpartnerInnen ist in Anhang 1 zu finden.

${ }^{4}$ Die Leitfäden zu den Workshops sind in Anhang 2 zu finden.

${ }^{5}$ Der Interviewleitfaden ist in Anhang 3 zu finden.
} 
Zudem wurde ein Usability-Test der Young Science-Webseite von vier Expertinnen am ZSI durchgeführt. ${ }^{6}$

Im Angebot des ZSI an das BMWFW war zudem eine Online-Konsultation mit SchülerInnen vorgesehen. In Absprache mit Frau Dr. Céline Loibl wurde diese Aktivität durch die erwähnten leitfadengestützten Interviews mit StakeholderInnen an Schulen und Universitäten ersetzt.

\section{Ergebnisse, Schlussfolgerungen \& Empfehlungen}

In der Datenerhebung für die Evaluierung von Young Science wurden zwei Schwerpunkte gesetzt:

1) Vernetzungsaktivitäten (Beratung, Veranstaltungen, Webseite, Newsletter und Facebook-Seite) und

2) von Young Science durchgeführte Projekte und Initiativen (Projekte im Themenschwerpunkt Nachhaltigkeit, Themenplattform für vorwissenschaftliche Arbeiten, Young Science Gütesiegel). Vorliegender Bericht folgt derselben Logik. Die Ergebnisse werden pro erwähnte Aktivität dargestellt, Schlussfolgerungen werden gezogen und Empfehlungen abgeleitet. Schließlich folgt ein kurzer Exkurs zu zukünftigen Aktivitäten von Young Science im Bereich Citizen Science.

\section{Vernetzung}

Das qualitative Feedback von Personen, die in enger Verbindung mit Young Science stehen und im Zuge von Interviews und einem Workshop befragt wurden, macht Gemeinsamkeiten und Unterschiede bei der Vernetzung verschiedener Zielgruppen deutlich.

Eine Gemeinsamkeit von Young Science-Kontaktpersonen an Universitäten, ForscherInnen und LehrerInnen ist, dass der Erstkontakt zu Young Science überwiegend projektspezifisch entstanden ist. Häufig stand ein Sparkling Science-Projekt am Beginn der Zusammenarbeit. In manchen Fällen kam der Erstkontakt auch über Young Science Projekte zum Thema Nachhaltigkeit zu Stande.

Einigkeit bestand - unabhängig von der Zielgruppe - darin, dass Young Science eine wichtige Rolle im Sinne der Koordination von Aktivitäten und Initiativen einnimmt. Das muss vor dem Hintergrund gesehen werden, dass ein Mangel an zeitlichen und finanziellen Ressourcen sowohl von Personen an Bildungseinrichtungen im sekundären als auch im tertiären Bereich als größte Barriere für die Zusammenarbeit von Schule und Wissenschaft genannt wurde. Daher wird eine Organisation, die gezielt vernetzt und Anstöße für neue Initiativen gibt, als sehr wertvoll wahrgenommen. Dabei kommt auch der Abstimmung mit anderen Initiativen große Bedeutung zu, da Doppelgleisigkeiten, eben vor dem Hintergrund beschränkter zeitlicher Ressourcen, schnell als abschreckend wahrgenommen werden. ${ }^{7}$

\section{- LehrerInnen}

Bei der Einbindung von Lehrpersonen in das Young Science-Netzwerk zeigten der Workshop und die Interviews, dass unflexible schulische Rahmenbedingungen eine Herausforderung darstellen. Nur in wenigen Fällen wurden berichtet, dass Projekte in den Regelunterricht integriert werden konnten. Eher werden sie im Rahmen von Freifächern - und auch diese müssen mit großer Vorlaufzeit angekündigt werden ${ }^{8}$ - angeboten, oder SchülerInnen engagieren sich unabhängig von Freifächern in

\footnotetext{
${ }^{6}$ siehe Anhang 4

${ }^{7}$ In Zusammenhang mit der Themenplattform wird darauf noch detaillierter eingegangen.

${ }^{8}$ Laut einem Direktor müssen unverbindliche Übungen ca. zwei Jahre im Voraus feststehen.
} 
ihrer Freizeit in Projekten. LehrerInnen müssen im Fall von Veranstaltungsteilnahmen um Freistellung und teilweise auch um die Abgeltung der Reisekosten ansuchen.

Das Gelingen einer Zusammenarbeit hängt stark vom persönlichen Engagement motivierter Lehrkräfte ab. Dieses Engagement außerhalb der regulären Unterrichtszeiten wird laut LehrerInnen häufig nicht abgegolten, wenngleich in diesem Zusammenhang anzumerken ist, dass beispielsweise bei Sparkling Science-Projekten sehr wohl die Möglichkeit zur Abgeltung dieses Engagements besteht. ${ }^{9}$ LehrerInnen, die Projekte an ihren Schulen umgesetzt haben, nennen Interesse am Thema und persönliche Weiterbildung als Motive für ihr Einbringen.

Wie die Erfahrung der Young Science-MitarbeiterInnen zeigt, sind LehrerInnen jedoch häufig schwer zu motivieren. Am leichtesten gelänge das über spezifische Projektarbeit an Schulen mit einem Forschungsschwerpunkt. Das qualitative Feedback aus dem Workshop und den Interviews zeigt, dass diese Schulen einen konkreten Nutzen in der Kooperation mit wissenschaftlichen Einrichtungen sehen. Einerseits werden solche Projekte als Möglichkeit zur Förderung besonders begabter SchülerInnen gesehen. Andererseits eignen sie sich in der Außendarstellung der Schulen für die Anwerbung neuer SchülerInnen.

\section{- SchülerInnen}

SchülerInnen werden von Young Science zumeist nur indirekt über Lehrpersonen einbezogen. ${ }^{10}$ Das Gelingen ihrer Einbindung hängt daher stark von der Einbindung von Lehrpersonen ab. Über die Themenplattform wird zukünftig der direkte Kontakt zu SchülerInnen verstärkt werden, da diese unabhängig Themen recherchieren und Fragen an Young Science stellen können.

\section{- Forscherlnnen}

Zeitliche Ressourcen sind auch bei der Vernetzung mit ForscherInnen ausschlaggebend. Die Erfahrungen von Young Science-Kontaktpersonen an Universitäten zeigen, dass der Fokus der WissenschafterInnen stark auf der (Grundlagen-)Forschung liegt. Trotzdem gibt es viele engagierte ForscherInnen, die - häufig auch in ihrer Freizeit - Aktivitäten für SchülerInnen organisieren (beispielsweise Führungen oder Exkursionen). Das spiegelt sich auch in der Erfahrung der Young Science-MitarbeiterInnen wieder. ForscherInnen können vor allem über Projekte, in denen Drittmittel zur Verfügung gestellt werden, in das Netzwerk eingebunden werden.

WissenschafterInnen wenden sich aber auch an Young Science, wenn sie für diverse Forschungsprojekte Kontakte zu Schulen benötigen. Besteht einmal Kontakt zu einer Forscherin/ einem Forscher über ein Projekt, bleiben diese ForscherInnen Young Science auch nach dem Projektende treu und stehen beispielsweise für Inputs bei Veranstaltungen zur Verfügung (mehr dazu im Kapitel „Beratung \& persönliche Kontaktanbahnung“).

\section{- Young Science-Kontaktpersonen}

Die Aussagen von Young Science-Kontaktpersonen an Universitäten verdeutlichen, dass an Hochschulen häufig Unklarheiten hinsichtlich der Aufgaben und Zuständigkeiten von Personen in dieser Rolle herrscht. Die Ergebnisse des Workshops und der Interviews weisen darauf hin, dass bei der Etablierung der Kontaktpersonen an Universitäten im Zuge der Leistungsvereinbarung nicht klar war, welche Erwartungen mit dieser Rolle verbunden sind. Befragte Kontaktpersonen gaben an, dass

\footnotetext{
${ }^{9}$ Sparkling Science (-): FAQs - Frequently Asked Questions, S. 3, http://www.sparklingscience.at/ downloads/FAQs.pdf.

${ }^{10}$ Eine Ausnahme stellt seit 2014 die Themenplattform für vorwissenschaftliche Arbeiten dar.
} 
dies nur einer ihrer Aufgabenbereiche ist und die zeitlichen Ressourcen für diese Aufgaben stark beschränkt sind. Young Science-MitarbeiterInnen verweisen zudem darauf, dass in großen Institutionen je nach Anliegen auch verschiedene Organisationseinheiten von Bedeutung sind (beispielsweise VizerektorInnen für strategische Fragen, Abteilungen für Öffentlichkeitsarbeit in Zusammenhang mit organisatorischen Belangen, etc.).

Für das Gelingen ihrer Arbeit sehen Kontaktpersonen ihre gute Vernetzung innerhalb der eigenen Institution als entscheidend an. Denn vor dem Hintergrund beschränkter zeitlicher Ressourcen reagieren ForscherInnen vor allem dann auf Anfragen, wenn ein persönlicher Bezug zur Person besteht.

Young Science-Kontaktpersonen wird die Durchsetzung ihrer Anliegen erleichtert, wenn eine Anfrage oder ein Auftrag von Young Science vorliegt. Es wurde wiederholt darauf verwiesen, dass dann eine klare Verbindung zum BMWFW gesehen und der Anfrage/dem Auftrag damit mehr Wichtigkeit beigemessen wird.

Diese zielgruppenspezifischen Erkenntnisse sind bei der Einbindung der verschiedenen Zielgruppen in das Young Science-Netzwerk bedeutend und spiegeln sich daher in den Empfehlungen zu den einzelnen Vernetzungsaktivitäten wider (diese sind jeweils am Ende der einzelnen Kapitel zu finden).

\section{Beratung \& aktive Kontaktanbahnung}

Von den verschiedenen Services, die von Young Science angeboten werden, wird vor allem der persönliche Kontakt sehr geschätzt. Bei Fragen oder Problemen einfach anrufen oder ein E-Mail schreiben zu können, erspare Zeit und wird der Recherche auf der Homepage häufig vorgezogen. Der persönliche Kontakt mit MitarbeiterInnen von Young Science wurde als unkompliziert wahrgenommen und die prompten Antworten sehr positiv hervorgehoben.

Zwei Kontaktpersonen an Universitäten merkten auch an, dass sie sich regelmäßig mit Petra Siegele treffen, wenn diese in Innsbruck bzw. Graz ist. Das wird als effizienter Weg wahrgenommen, um auf dem Laufenden zu bleiben.

Bedarf für regelmäßige Sprechstunden wurde hingegen keiner gesehen. Dem stünde der hohe Zeitaufwand entgegen, da die meisten Anliegen auch telefonisch geklärt werden können. Im Bedarfsfall wurde zudem die Möglichkeit erwähnt, sich einfach persönlich einen Termin vereinbaren zu können.

Dass ein Anruf oder ein E-Mail der Konsultation der Webseite vorgezogen wird, könnte auch damit zusammen hängen, dass die interviewten Personen und Workshop-TeilnehmerInnen eng mit Young Science in Verbindung stehen. Für Personen, die mit Young Science und den dort arbeitenden Personen weniger vertraut sind, ist die Hemmschwelle anzurufen möglicherweise höher. Für jene Personen ist die Webseite als erste Anlaufstelle ein wichtiger Service. Die steigenden BesucherInnenZahlen auf der Webseite weisen darauf hin (siehe Kapitel „Webseite und Facebook-Seite“). Auch Young Science-MitarbeiterInnen bestätigen, dass der persönliche Kontakt von großer Bedeutung ist. Dies betrifft vor allem die Einbindung neuer AkteurInnen in das Netzwerk. Ein persönliches Gespräch sei gerade bei Personen wichtig, die Young Science noch nicht kennen, um sie über die Angebote zu informieren. Laut Young Science-MitarbeiterInnen sei das Erreichen neuer Personen über den Newsletter schwierig. Auch hier dürfte die bereits erwähnte Zeitknappheit der verschiedenen AkteurInnen eine Rolle spielen. Wie oben erwähnt, ist ein konkretes Anliegen oder Angebot in der Form eines Projektes hier eher zielführend. 
In den vergangenen Jahren konnte auch das im Angebot formulierte Ziel erreicht werden, dass neben Universitäten und Fachhochschulen zunehmend Schulen Kontaktpersonen für die Forschungs-

Bildungs-Kooperation nominieren. Dabei handelt es sich nicht ausschließlich um mit dem Gütesiegel prämierte Schulen.

Die Etablierung von Kontaktpersonen an Pädagogischen Hochschulen war bislang im Vergleich zu Universitäten und Fachhochschulen wenig erfolgreich. Die Ursache dafür wird in der fehlenden Zuständigkeit des BMWFW für diesen Ausbildungszweig gesehen, wodurch die Etablierung von Young Science-Kontaktpersonen nicht im Zuge der Leistungsvereinbarung angebahnt werden kann.

\section{Empfehlungen:}

- Da die persönliche Kontaktebene von den Befragten als äußerst wichtig hervorgehoben wurde, sollten „aufsuchende“ Informationsangebote ${ }^{11}$ weiter ausgebaut werden (siehe auch Empfehlungen zu Veranstaltungen).

- Anstrengungen zur Etablierung von Young Science-Kontaktpersonen an den Pädagogischen Hochschulen sollten forciert werden. Dies würde zu einer stärkeren Öffnung von Young Science gegenüber Schultypen wie Neuen Mittelschulen und Volksschulen ${ }^{12}$ beitragen. Gleichzeitig könnten angehende Lehrerlnnen noch während ihrer Ausbildung für die Forschungs-Bildungs-Kooperation interessiert werden. Die Etablierung solcher Kontaktstellen könnte in Zusammenarbeit mit dem BMBF angebahnt werden, da das direkte Kontaktieren einzelner Pädagogische Hochschulen seitens Young Science bisher wenig erfolgreich war.

- Teilweise scheint es bei den Young Science-Kontaktpersonen Unklarheiten bezüglich ihrer Rolle zu geben. Young Science könnte dazu beitragen, gemeinsam mit den Kontaktpersonen ihr Rollenprofil zu schärfen.

- Laut Young Science-MitarbeiterInnen wurden Gespräche mit Kontaktpersonen an Universitäten zuletzt verstärkt in einer größeren Runde geführt (z.B. mit Einbindung der Abteilungen für Öffentlichkeitsarbeit). Dies scheint ein wichtiger Ansatzpunkt zu sein, um verschiedene Organisationseinheiten, die in ihrer Arbeit Kontakt zu Schulen haben, auf den gleichen Wissensstand zu bringen. Solche Gespräche sollten zukünftig verstärkt durchgeführt werden (etwa in Zusammenhang mit konkreten Projekten wie der Themenplattform ${ }^{13}$ ) und können dazu beitragen, die Rolle von Young Science-Kontaktpersonen innerhalb ihrer Institution zu stärken.

\section{Veranstaltungen}

Durch die Organisation eigener Veranstaltungen verfolgt Young Science das Ziel, Informationsaustausch und Vernetzung zu fördern.

Laut Anbot des OeAD waren Informationsveranstaltungen und Veranstaltungen zum Erfahrungsaustausch und zur Weiterentwicklung des Netzwerkes geplant. Bei den Veranstaltungen, die in Form von Workshops durchgeführt werden, sollten rund 20 Personen teilnehmen. Bei den Diskussionsrunden und Vorträgen wurden 30 bis 50 TeilnehmerInnen angestrebt. Neben der alljährlichen Young Science Tagung waren drei bis vier kleinere Veranstaltungen pro Jahr vorgesehen.

\footnotetext{
${ }^{11}$ Darunter sind Angebote zu verstehen, bei denen an Orten aktiv auf potentielle AkteurInnen zugegangen wird, an denen diese üblicherweise anzutreffen sind (z.B. bei Weiterbildungsveranstaltungen für BildungsberaterInnen, auf Tagungen von DirektorInnen, „BotschafterInnen-Ansatz“ in Schulen).

${ }^{12}$ siehe Kapitel zum Gütesiegel

${ }^{13}$ Wie sich im Workshop und bei den Interviews zeigte, dürfte es bezüglich vorwissenschaftlichen Arbeiten großes Unwissen an den tertiären Bildungseinrichtungen geben.
} 
Zielgruppen der Veranstaltungen waren SchulleiterInnen, Bildungs- und Studienberaterlnnen, MitarbeiterInnen von Universitäten und WissenschafterInnen.

Die Evaluierung zeigt, dass die Anzahl der in den letzten Jahren durchgeführten Veranstaltungen höher war als im Angebot geplant. Ende 2011 fand ein erstes Netzwerktreffen statt. 2012 gab es insgesamt fünf Veranstaltungen (darunter zwei Netzwerktreffen), im Jahr 2013 sechs (darunter vier Netzwerktreffen) und im Jahr 2014 neun (darunter ein Netzwerktreffen).

Die Anzahl der Personen, die über Veranstaltungen erreicht wurde, ist seit 2011 deutlich gestiegen (siehe Grafik 1). Der leichte Einbruch im Jahr 2013 erklärt sich daraus, dass in diesem Jahr deutlich mehr Netzwerktreffen stattfanden als in den anderen Jahren. Diese Veranstaltungen werden in einem kleineren Format durchgeführt.

Mit durchschnittlich 132 Personen pro Veranstaltung seit 2011 wurde die im Angebot formulierte TeilnehmerInnenzahl deutlich übertroffen.

Grafik 1: VeranstaltungsteilnehmerInnen gesamt nach Jahr

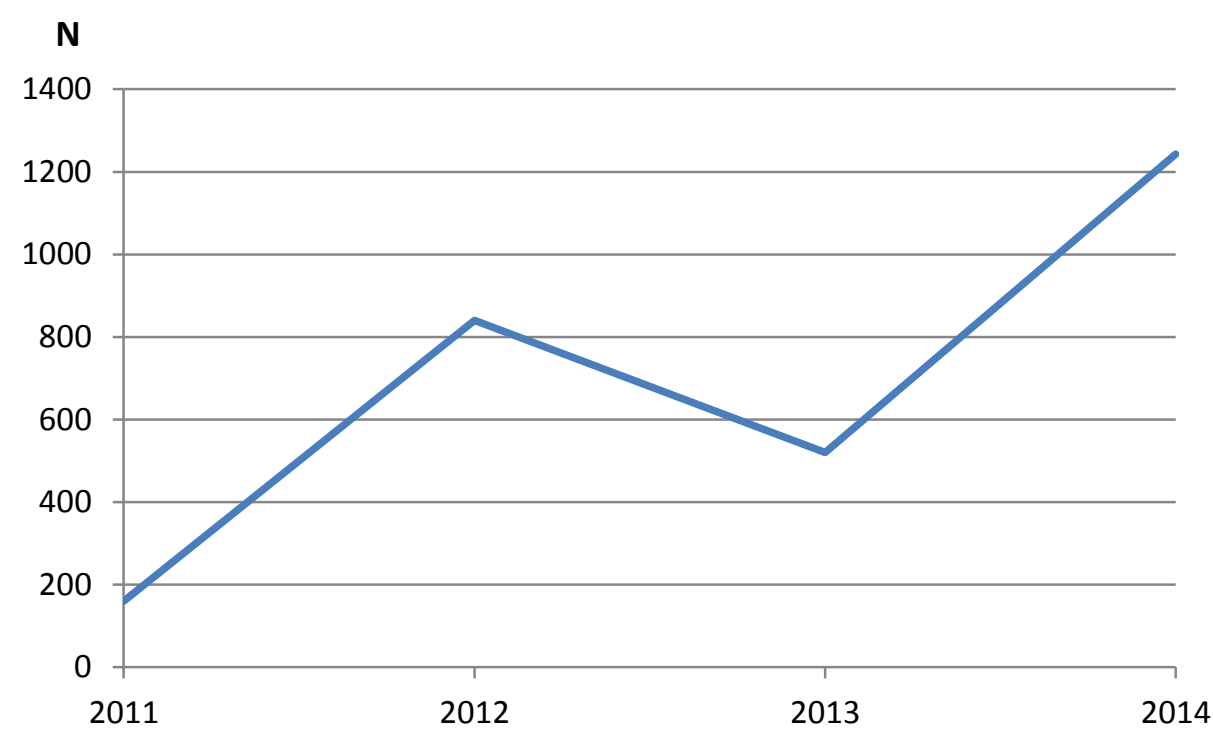

Die untenstehende Grafik schlüsselt die TeilnehmerInnen der Veranstaltungen nach Zielgruppen auf. Die Einbindung von WissenschafterInnen lag in den letzten drei Jahren bei rund 200 und war damit weitgehend konstant. Die Teilnahme von Lehrpersonen schwankte seit 2012 stärker (zwischen 121 und 281 Personen) und war im Jahr 2013 niedrig. Ein starker Anstieg ist hingegen bei der Teilnahme von SchülerInnen zu beobachten. 2014 waren SchülerInnen über verschiedene (neue) Formate, wie die Science Slams und den Sparkling Science-Kongress stark involviert (507 Schülerlnnen im Jahr 2014). Bei „Interessierten“ handelt es sich laut einem/einer MitarbeiterIn von Young Science um Personen aus Ministerien oder ähnlichen Einrichtungen, wie der Wirtschaftskammer oder der FGG, sowie um Initiativen, die an der Schnittstelle zwischen Wissenschaft und Schule tätig sind. Auch bei dieser Zielgruppe ist im Jahr 2014 ein Zuwachs bei der Veranstaltungsteilnahme zu verzeichnen. 


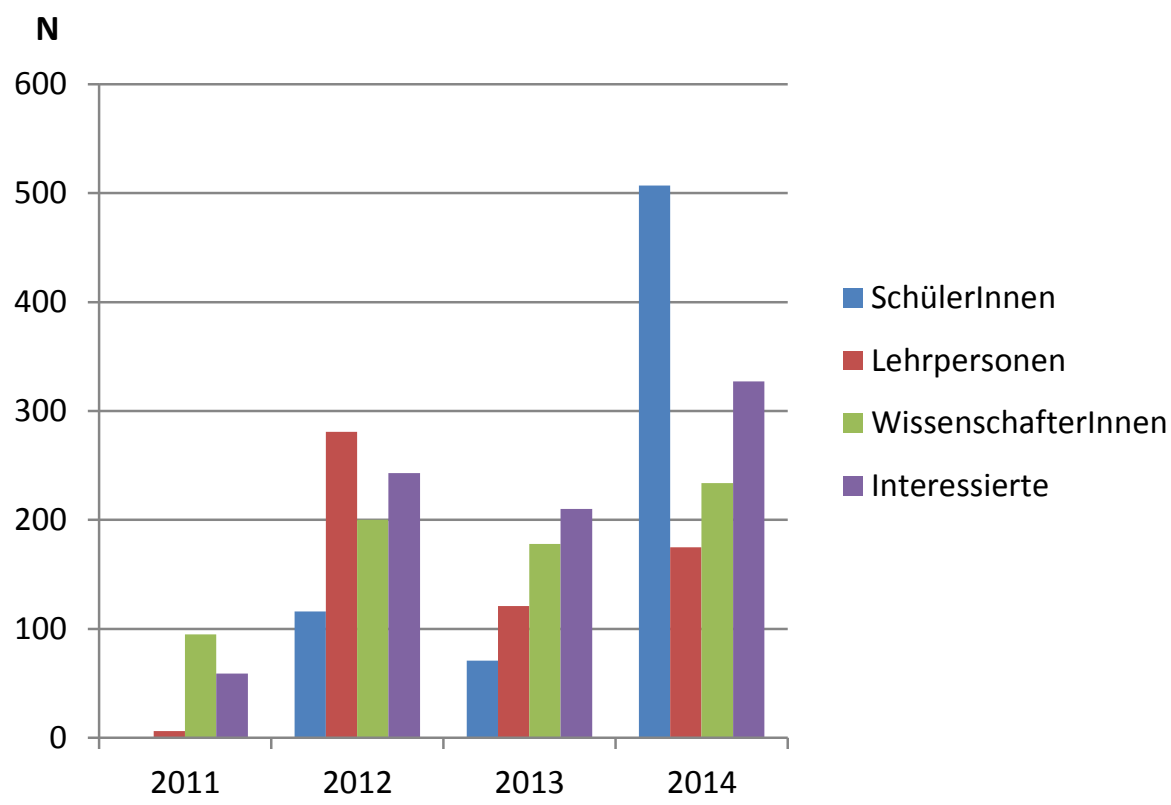

Eine weitere wichtige Aktivität von Young Science in Zusammenhang mit Veranstaltungen stellen Inputs im Zuge von bereits bestehenden Veranstaltungen für Schüler- und BildungsberaterInnen dar. In diesem Rahmen werden bestehende Veranstaltungen genutzt, um über die Aktivitäten und Services von Young Science zu informieren. Im Jahr 2014 ist zu beobachten, dass diese Aktivitäten auch auf die Zielgruppe der DirektorInnen ausgeweitet wurden. ${ }^{14}$

Seit 2011 haben 18 solcher Inputs stattgefunden - mit stetig steigender Anzahl pro Jahr (siehe Grafik 3).

Grafik 3: Anzahl der Inputs bei Veranstaltungen für Schüler-/BildunsberaterInnen, DirektorInnen etc. nach Jahr

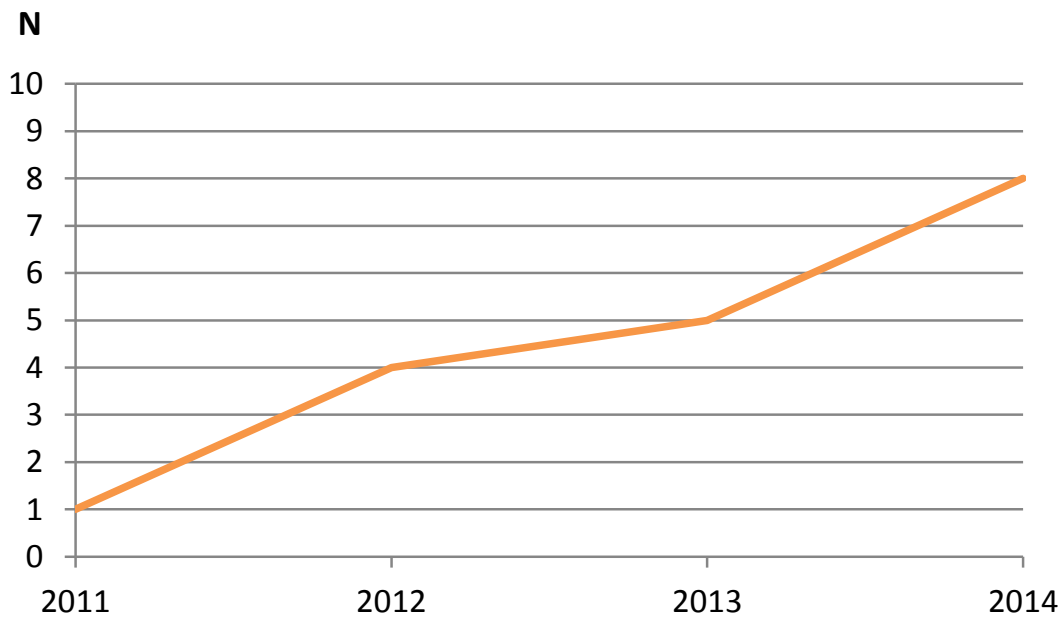

Wie untenstehende Tabelle zeigt, sind diese Inputs - trotz einer leichten Konzentration im Osten des Landes - gleichmäßiger über ganz Österreich verteilt, als von Young Science selbst organisierte Veranstaltungen. Bei Young Science-Veranstaltungen dominiert Wien als Veranstaltungsort. Einzelne

\footnotetext{
${ }^{14}$ Inputs wurden bei Treffen von DirektorInnen in Kärnten, Niederösterreich, Tirol und dem Burgenland gegeben.
} 
Young Science-Veranstaltungen fanden aber auch in den größeren Landeshauptstädten statt (Graz, Innsbruck, Salzburg, St. Pölten).

Tabelle 1: Anzahl der Veranstaltungen nach Bundesland

\begin{tabular}{|lrrr|} 
& $\begin{array}{c}\text { Anzahl der "Infotour" } \\
\text { Veranstaltungen }\end{array}$ & $\begin{array}{c}\text { Anzahl der YS- } \\
\text { Veranstaltungen }\end{array}$ & 0 \\
\hline Burgenland & 3 & 0 & Gesamt \\
\hline Kärnten & 1 & 0 & 3 \\
\hline Oberösterreich & 1 & 1 & 1 \\
\hline Niederösterreich & 4 & 1 & 1 \\
\hline Salzburg & 2 & 2 & 5 \\
\hline Steiermark & 2 & 2 & 3 \\
\hline Tirol & 2 & 0 & 4 \\
\hline Vorarlberg & 1 & 15 & 4 \\
\hline Wien & 2 & $\mathbf{2 1}$ & 1 \\
\hline Gesamt & $\mathbf{1 8}$ & & 17 \\
\hline
\end{tabular}

Von insgesamt 21 Young Science-Veranstaltungen in den Jahren 2011-2014 wurden 16 Veranstaltungen mittels TeilnehmerInnenbefragung evaluiert. Die Ergebnisse pro Veranstaltung wurden dem ZSI zur weiteren Analyse zur Verfügung gestellt.

Da im Jahr 2014 die Skala des Fragebogens geändert wurde, sind die Ergebnisse für das Jahr 2014 gesondert dargestellt. Durch die unterschiedlichen Skalen lassen sich die Ergebnisse der Analysen von 2011-2013 und von 2014 nicht direkt miteinander vergleichen.

Von 2011 bis 2013 wurden sieben von zwölf (58\%) Veranstaltungen evaluiert. Der Rücklauf bei der TeilnehmerInnenbefragung lag bei durchschnittlich 19\%. Aufgrund dieser geringen Rücklaufquote lassen sich die Ergebnisse nicht auf alle Teilnehmerlnnen umlegen.

Tabelle 2: Durchschnittliche Bewertung der Veranstaltungen 2011-2013 durch die TeilnehmerInnen

\begin{tabular}{|lr|}
\multicolumn{1}{|l|}{$\left(\begin{array}{r}\text { durchschnittliche Bewertung } \\
\text { Erreichbarkeit }\left(\mathrm{N}^{15}=\mathbf{7}\right)\end{array}\right.$} & 1,2 \\
\hline Atmosphäre $(\mathrm{N}=7)$ & 1,7 \\
\hline Vorinformationen $(\mathrm{N}=7)$ & 1,6 \\
\hline Betreuung vor Ort $(\mathrm{N}=7)$ & 1,5 \\
\hline Programm ${ }^{16}(\mathrm{~N}=7)$ & 1,6 \\
\hline Moderation $(\mathrm{N}=6)^{2}$ & 1,4 \\
\hline Verpflegung $(\mathrm{N}=7)$ & 1,4 \\
\hline
\end{tabular}

Im Jahr 2014 wurden alle neun Veranstaltungen evaluiert. Der Rücklauf bei der TeilnehmerInnenbefragung lag bei durchschnittlich 34\% und war damit etwas höher als in den vorangegangenen Jahren.

\footnotetext{
${ }^{15}$ Anzahl der analysierten Veranstaltungen

${ }^{16}$ Es gab im Fragebogen mehrere Fragen zu den einzelnen Programmpunkten. Über diese Fragen wurde pro Veranstaltung ein Mittelwert gebildet.
} 
Tabelle 3: Durchschnittliche Bewertung der Veranstaltungen 2014 durch die TeilnehmerInnen

durchschnittliche Bewertung

(1=sehr gut, 2=gut, 3 =eher schlecht, 4=schlecht)

\begin{tabular}{|lr|}
\hline Erreichbarkeit $(\mathrm{N}=9)$ & 1,3 \\
\hline Atmosphäre $(\mathrm{N}=8)$ & 1,3 \\
\hline Vorinformationen $(\mathrm{N}=9)$ & 1,4 \\
\hline Betreuung vor Ort $(\mathrm{N}=8)$ & 1,2 \\
\hline Programm $(\mathrm{N}=7)$ & 1,6 \\
\hline Moderation $(\mathrm{N}=4)$ & 1,5 \\
\hline Verpflegung $(\mathrm{N}=8)$ & 1,3 \\
\hline
\end{tabular}

Bei den Veranstaltungen von 2011 bis 2013 wurde die Erreichbarkeit durchschnittlich am besten bewertet, gefolgt von der Moderation und der Verpflegung. Auch im Jahr 2014 war die Bewertung der Erreichbarkeit und der Verpflegung sehr positiv. Die durchschnittlich beste Bewertung bei den Veranstaltungen 2014 bekam allerdings die Betreuung vor Ort.

2014 wurde das Programm durchschnittlich am schlechtesten bewertet. Auch in den Jahren 20112013 war die Bewertung des Programms im Vergleich zu anderen Aspekten nicht besonders gut (zweitschlechteste Bewertung).

Generell zeigt sich bei der Analyse des Feedbacks der VeranstaltungsteilnehmerInnen jedoch ein positives Bild, da alle Ergebnisse in der positiven Hälfte der Skalen liegen.

Als wertvolle Veranstaltungen wurden im Workshop und bei den Interviews besonders die Vernetzungstreffen der Kinderuniversitäten, die sich gezielt an OrganisatorInnen von Kinderuniversitäten richten, hervorgehoben. Bei diesen AkteurInnen bestand ein klarer Bedarf an Austausch, aber es fehlte ein Mittler, der den Austausch organisiert und strukturiert. Die Personen, die Kinderuniversitäten organisieren, hatten sich zuvor vielfach gegenseitig nicht gekannt. Eine Person merkte an, dass dieser Austausch auch zur Weiterentwicklung der Kinderuniversitäten beigetragen habe und neue Kooperationen entstanden sind. Auch ein/e Mitarbeiterln von Young Science berichtete, dass beim zweiten Austauschtreffen bereits über gemeinsame Projekte nachgedacht wurde.

Sehr positiv war auch das Feedback zur Infotour, bei der im Rahmen anderer Veranstaltungen das Angebot von Young Science präsentiert wird. Dabei wurde vor allem der Aspekt, regelmäßig etwas in Erinnerung zu rufen und auf dem Laufenden zu bleiben, ohne eigeninitiativ werden zu müssen, hervorgehoben.

Die Einbindung von SchülerInnen bei Veranstaltungen wurde von den LehrerInnen sehr positiv aufgenommen. Veranstaltungen würden es den SchülerInnen ermöglichen „über den Tellerrand zu blicken“, indem sie z.B. Präsentationen anderer Schulen sehen und so Einblick in neue Themen- und Forschungsfelder bekommen. Der Besuch von Veranstaltungen sei für SchülerInnen etwas Besonderes und eine Art Anerkennung ihrer Leistungen. Diese Aussage muss auch vor dem Hintergrund beurteilt werden, dass SchülerInnen oft im Rahmen von Freifächern und in ihrer Freizeit in Projekten mitarbeiten.

Bei der Einbindung von SchülerInnen in Veranstaltungen müsse immer beachtet werden, dass das Programm sehr genau auf SchülerInnen und die jeweilige Altersgruppe zugeschnitten ist und die Veranstaltungen (wenn möglich) in den Bundesländern stattfinden, um die Anreise zu erleichtern. Der Science Slam und die Workshops für die GewinnerInnen der Science Slam-Vorentscheidungen 
wurden - mit Verweis auf das abwechslungsreiche Programm und den Austausch von Schülerlnnen untereinander - als positives Beispiel erwähnt.

Zeitliche Ressourcen werden sowohl von Lehrpersonen als auch von Kontaktstellen an Universitäten als Barriere für die Veranstaltungsteilnahme genannt. Bei Lehrpersonen kommen zudem die benötigte Freistellung und das komplizierte Prozedere bei der Reisekostenübernahme ${ }^{17}$ hinzu. Dass Veranstaltungen häufig in Wien stattfinden, wurde von Personen, die aus anderen Bundesländern anreisen müssen, wiederholt negativ angemerkt.

Aus den Ergebnissen lässt sich schließen, dass Veranstaltungen gerade dann gut angenommen werden, wenn sie zielgruppenspezifisch gestaltet sind und ein konkretes Thema behandeln. Der Nutzen muss für die TeilnehmerInnen schon vor der Veranstaltung ersichtlich sein, damit trotz geringer zeitlicher Ressourcen eine Anreise nach Wien angedacht wird (beispielhaft hierfür sind die Vernetzungsveranstaltungen für Kinderuniversitäten).

\section{Empfehlungen:}

- Aufgrund der Ergebnisse von Workshops und Interviews empfiehlt es sich, zielgruppen- und themenspezifische Veranstaltungen anzubieten (beispielsweise Management von Schulprojekten für LehrerInnen). ${ }^{18}$ Auch wenn dies zu Veranstaltungen in kleinerem Rahmen führen könnte, wäre dennoch der Mehrwert für die TeilnehmerInnen klarer. Solche Veranstaltungen könnten auch leichter vor Ort in den Bundesländern angeboten werden.

- Aufgrund des positiven Feedbacks zu Präsentationen von Young Science im Rahmen anderer Veranstaltungen (Infotour), sollte der Input von Young Science als Fixpunkt bei bestehenden, wiederkehrenden Weiterbildungs- und Vernetzungsveranstaltungen (z.B.

DirektorInnentagungen) etabliert werden. Dadurch wäre der persönliche Zugang zu verschiedenen Zielgruppen gewährleistet.

- Da persönliche Beziehungen von allen Befragten als wichtig eingeschätzt werden und der „aufsuchende“ Ansatz, der im Rahmen der Infotour verfolgt wird, auf sehr positives Feedback stößt, scheint die Idee, in Zukunft ForscherInnen als BotschafterInnen an Schulen zu entsenden, vielversprechend. Zusätzlich sollte geprüft werden, ob dieser Ansatz sich auch für das Gewinnen neuer LehrerInnen für die Zusammenarbeit mit WissenschafterInnen eignet. Damit könnten LehrerInnen mit Erfahrung in der Kooperation mit wissenschaftlichen Einrichtungen an anderen Schulen über ihre Projekterfahrungen berichten.

- Bei sehr spezifischen Themen könnten virtuelle Meetings (z.B. Webinare) angedacht werden. Insbesondere ForscherInnen dürften aufgrund der starken Internationalisierung der Forschung damit bereits vertraut sein. Virtuelle Veranstaltungsformate würden helfen organisatorische und zeitliche Barrieren zu minimieren.

- Auch für die stärkere Einbindung für SchülerInnen könnten sich virtuelle Meetings eignen. ${ }^{19}$

\footnotetext{
${ }^{17}$ Eine Lehrperson berichtete, dass bei der Übernahme von Reisekosten ein Ansuchen beim Landesschulrat notwendig ist.

${ }^{18}$ Von einer Lehrperson kam im Zuge der Befragung etwa der Wunsch nach Tipps und Informationen zur praktischen Umsetzung von Projekten an Schulen.

${ }^{19}$ In einem Citizen Science Projekt (http://socientize.eu/), in das das ZSI involviert war, gab es beispielsweise positives Feedback zu Webkonferenzen von SchülerInnen mit ForscherInnen.
} 


\section{Webseite \& Facebook-Seite}

Die Young Science Webseite stellt einerseits eigene Projekte und Initiativen (wie das Young ScienceGütesiegel und die Themenplattform für vorwissenschaftliche Arbeiten) vor, andererseits bewirbt sie fremde Initiativen, wie Sparkling Science, das Projekt „Studieren probieren“ oder die MaturantInnenberatung. Somit wird versucht, ein Ziel des Young Science Zentrums, nämlich Informationen zu Bündeln und Synergien zwischen den Initiativen zu nutzen, zu erreichen. Im Aufbau orientiert sie sich an verschiedene Zielgruppen: SchülerInnen, LehrerInnen, SchülerberaterInnen und ForscherInnen.

Laut MitarbeiterInnen von Young Science schätzen BildungsberaterInnen an der Webseite vor allem die direkten Verlinkungen zu jenen Seiten von tertiären Bildungseinrichtungen, die für angehende Studierende von Interesse sind. Lehrpersonen greifen häufig auf die Kontaktdaten der Young Science-Kontaktpersonen an Universitäten und Fachhochschulen zurück. ForscherInnen besuchen die Webseite in Zusammenhang mit Sparkling Science-Ausschreibungen vermehrt, um sich über Erfahrungen von SchülerInnen, FoscherInnen und Lehrpersonen zu informieren.

Im Zuge dieser Evaluierung wurden die Webstatistiken der Young Science Webseite analysiert. Die Anzahl der UserInnen ist in den letzten Jahren deutlich gestiegen. Da die Webseite erst Ende 2011 online ging, ist die BesucherInnenzahl für 2011 mit ca. 1.000 Personen noch relativ gering. In den Jahren 2012 und 2013 lag sie jeweils bei ca. 10.000 Personen und stieg 2014 stark an (auf 24.000 Personen) (siehe Grafik 4). Der starke Anstieg könnte sich durch die Themenplattform und Aktivitäten in Zusammenhang mit der Themenakquisition bzw. der Bewerbung der Plattform erklären. Mit den zur Verfügung gestellten Daten kann das allerdings nicht überprüft werden.

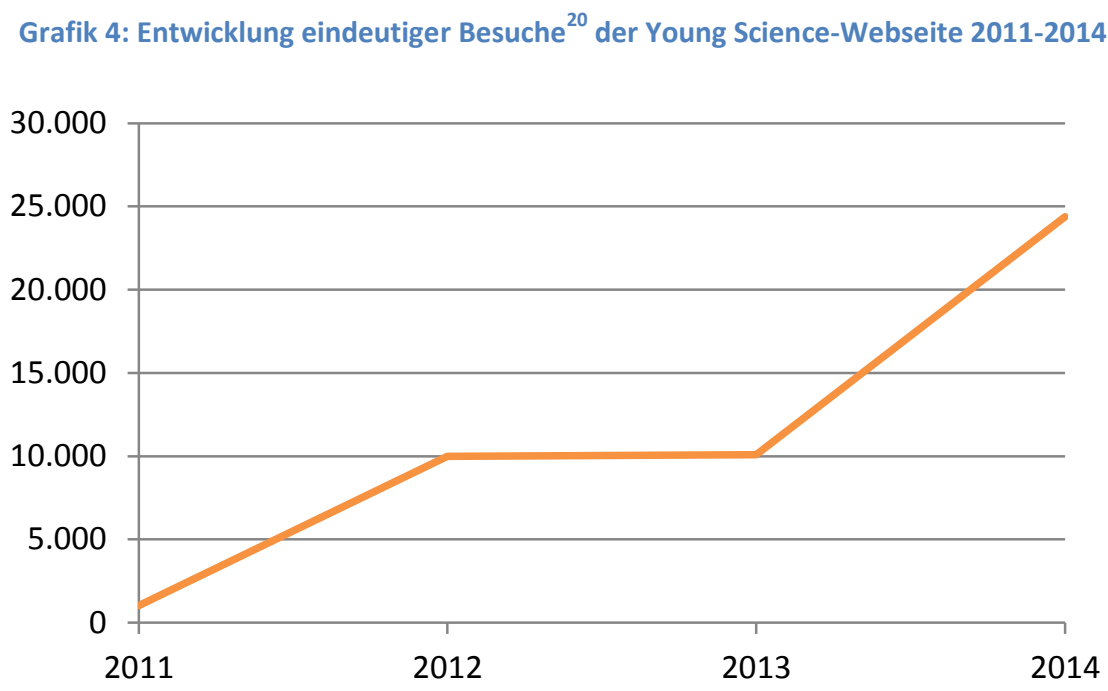

Die Absprungrate, also Personen, die die Webseite nach dem ersten Seitenaufruf wieder verlassen, lag in den vergangenen vier Jahren zwischen 38\% und 56\%. Im Jahr 2014 betrug sie 48\% und konnte somit im Vergleich zu den beiden Vorjahren gesenkt werden. Laut Google Analytics ${ }^{21}$ ist eine Absprungrate von $40-60 \%$ bei Webseiten zu bestimmten Inhalten ${ }^{22}$ durchaus üblich. ${ }^{23}$

\footnotetext{
${ }^{20}$ Anzahl der Personen, die die Webseite mindestens einmal aufgerufen haben.

${ }^{21}$ Google Analytics ist ein Service des Unternehmens Google, das zur Analyse von Webseitenstatistiken dient. Die Daten mehrerer hunderttausend Webseiten-Konten werden ausgewertet. Diese Auswertungen dienen als Referenzen.

${ }^{22}$ Im Unterschied zu Blogs, Serviceseiten oder Webseiten, die Produkte zum Verkauf anbieten.
} 


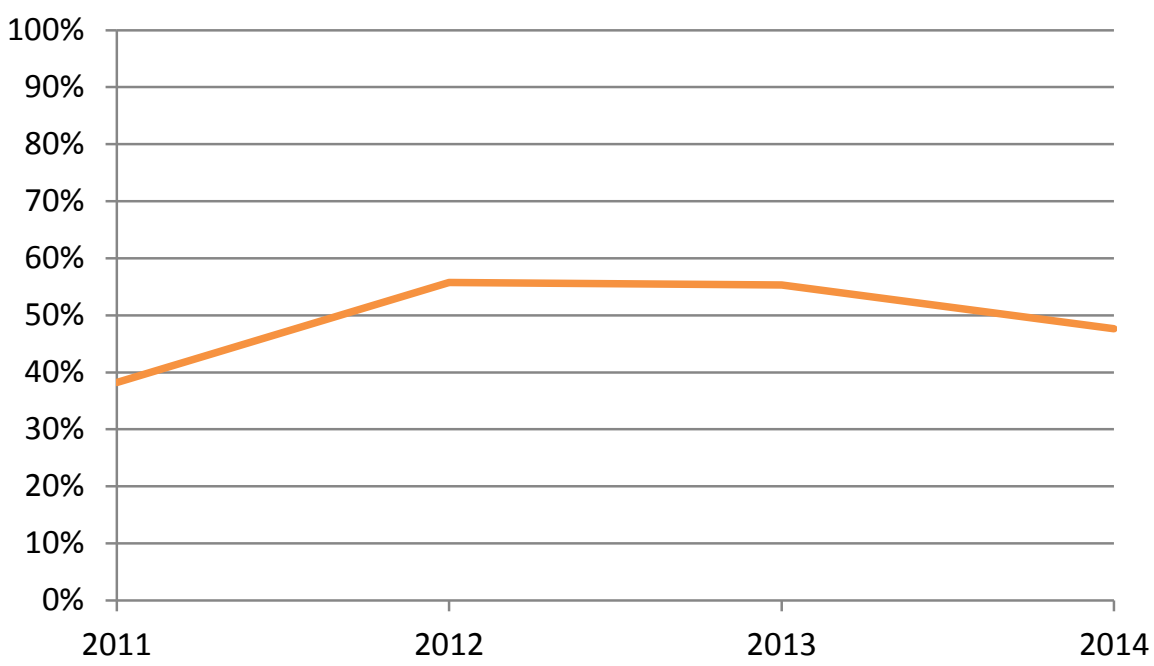

Neben der gestiegenen BesucherInnenzahl ist seit 2012 sowohl bei der Besuchsdauer als auch bei der durchschnittlichen Anzahl der aufgerufenen Seiten pro Besuch ein Anstieg zu verzeichnen. Im Jahr 2014 wurden erstmals die dokumentierten Werte der letzten beiden Monate im Jahr $2011^{25}$ überschritten. Im Jahr 2014 betrug die durchschnittliche Besuchsdauer auf der Young ScienceWebseite 4 Minuten und 20 Sekunden (siehe Tabelle 4). Als Vergleichswert dient der von Google Analytics im Jahr 2010/2011 gemessene Wert von 5 Minuten und 49 Sekunden, der somit um ca. 1,5 Minuten höher ist. Auch die durchschnittlich aufgerufene Seitenzahl hat im Jahr 2014 ihren vorläufigen Höhepunkt mit 4,8 Seiten erreicht (siehe Tabelle 5). Im Jahr 2010/2011 lag der von Google Analytics gemessene Durchschnittwert bei 4,9 Seiten pro Besuch. ${ }^{26}$ Er war damit fast ident mit dem 2014 gemessenen Wert der Young Science-Webseite.

Tabelle 4: Entwicklung der Besuchsdauer der Young Science-Webseite 2011-2014

\begin{tabular}{|lr|}
\hline 2011 & $\begin{array}{r}\text { durchschnittliche } \\
\text { Besuchsdauer }\end{array}$ \\
\hline 2012 & $00: 04: 03$ \\
\hline 2013 & $00: 02: 58$ \\
\hline 2014 & $00: 03: 31$ \\
\hline
\end{tabular}

\footnotetext{
${ }^{23}$ Quelle Referenzwert Absprungrate: http://yourwebsiteengineer.com/162-improve-websites-bounce-rate/

${ }^{24}$ Anteil an Webseitenbesuche mit nur einem einzelnen Seitenaufruf

${ }^{25}$ Die Seite ging im November 2011 online.

${ }^{26}$ Quelle Referenzwerte: https://blog.seibert-media.net/blog/2011/07/13/google-benchmark-wie-surfendeutsche-nutzer-internationaler-vergleich/
} 


\begin{tabular}{|lr|}
\hline \multicolumn{1}{|c}{} & $\begin{array}{r}\text { aufgerufene Seiten pro } \\
\text { Webseitenbesuch }\end{array}$ \\
\hline 2011 & 4,49 \\
\hline 2012 & 3,35 \\
\hline 2013 & 3,87 \\
\hline $\mathbf{2 0 1 4}$ & 4,79 \\
\hline
\end{tabular}

Die Referenzwerte bezüglich der Besuchsdauer und der Seitenaufrufe sind jedoch mit Vorsicht zu interpretiert, da darunter immer alle Typen von Webseiten subsumiert wurden (z.B. auch Blogs oder Verkaufsseiten). Und auch bei der Interpretation der bei der Young Science-Webseite gemessenen Werte muss mit Vorsicht vorgegangen werden, da eine kurze Besuchsdauer und eine geringe durchschnittliche Zahl an Seitenbesuchen auch darauf hinweisen könnten, dass die gesuchten Inhalte (z.B. Kontaktperson oder Liste von kooperationsinteressierten Schulen) von dem/der Besucherln schnell gefunden wurden. Mehr Aufschluss über die Usability der Webseite gibt daher der durchgeführte Test.

\section{Usability-Test der Young Science-Webseite}

Im Zuge dieser Evaluierung wurde ein Usability-Test der Young Science-Webseite am ZSI durchgeführt. Vier Usability-Expertinnen testeten anhand verschiedener Aufgaben (siehe Anhang 4) die Benutzerfreundlichkeit der Webseite.

Für den Usability-Test wurden fünf Aufgaben formuliert, in denen in unterschiedlichen Rollen (SchülerIn, LehrerIn, BildungsberaterIn, ForscherIn) verschiedene Informationen auf der Webseite gesucht werden sollten. Der Usability-Test wurde mittels Thinking-Aloud-Methode ${ }^{27}$ evaluiert. Die Testerinnen wurden dabei gebeten, ihre Gedanken zu verbalisieren, um Prozesse und Entscheidungen bei der Suche nach Informationen besser nachvollziehen zu können (z.B. in Hinblick auf "Warum klicke ich hier und nicht dort?", "Was erwarte ich mir in dieser Rubrik?"). Ausgangspunkt für jede Aufgabe war die Startseite der Website.

Die Testerinnen orientierten sich grundsätzlich an den unterschiedlichen Zielgruppen (z.B. Rubrik: Schüler/innen oder Lehrer/innen) auf der Startseite (siehe Abbildung 1).

\footnotetext{
${ }^{27}$ Eine kurze Methodenbeschreibung findet sich hier: http://www.bui.hawhamburg.de/pers/ursula.schulz/use/methoden/usability-tests.html
} 


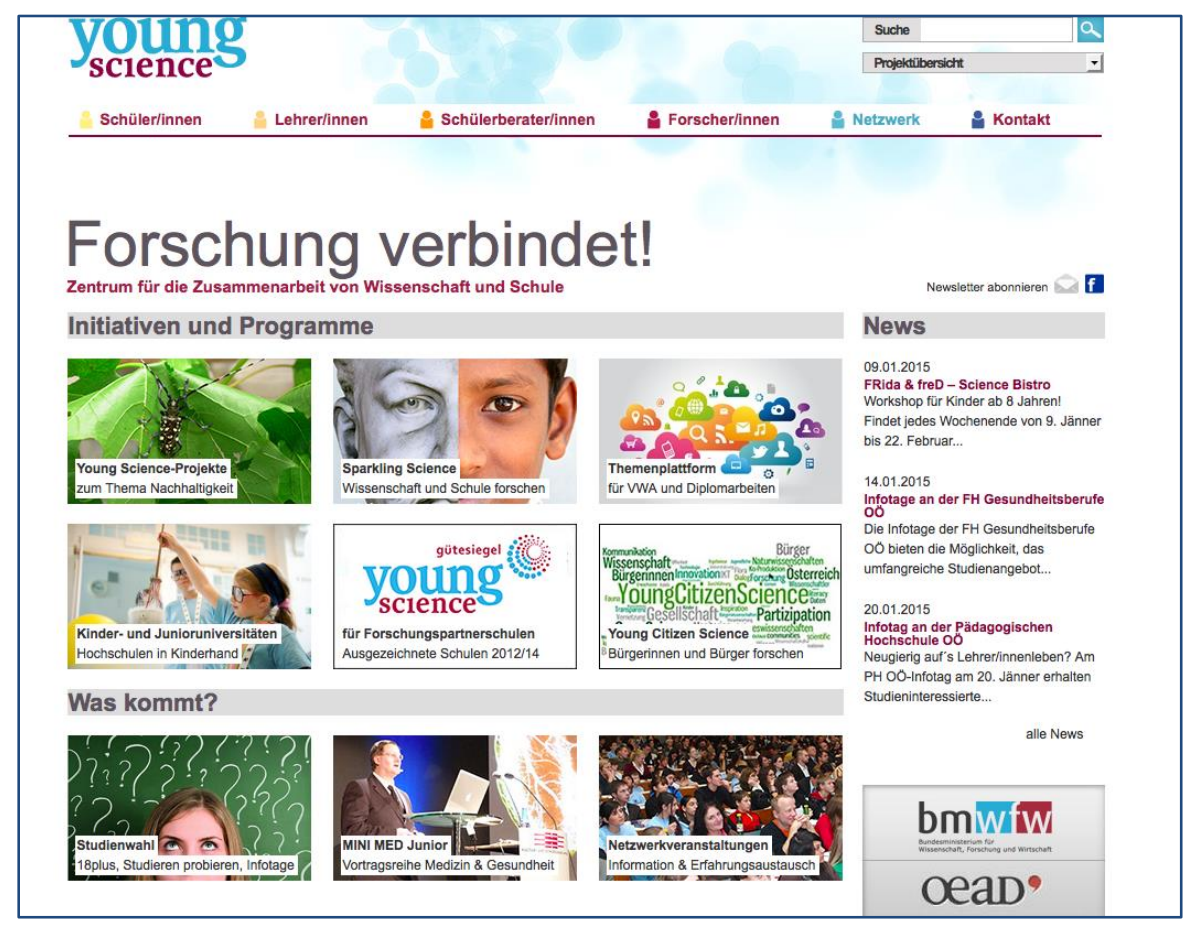

Abbildung 1: Screenshot der Startseite von Young Science (14.01.2014)

Die Aufteilung in die vier Zielgruppen: Schüler/innen, Lehrer/innen, Bildungsberater/innen und Forscher/innen erscheint sinnvoll, da - wie die Tests zeigen - Personen diese Rubriken als Informationssammlung für die eigene Zielgruppe verstehen „Ich als Schülerin suche bei Schüler/innen“.

Der Usability-Test hat aber auch gezeigt, dass nicht jede der gesuchten Informationen mit den relevanten Zielgruppen verlinkt ist. Zum Beispiel war die Suche nach Erfahrungsberichten von Schulen über ihre Mitarbeit in Projekten für die meisten Testerinnen schwierig zu finden, da die Information weder mit der Rubrik Schüler/innen noch bei jener der Lehrer/innen verlinkt ist, sondern bei Forscher/innen zu finden ist, die Testerinnen aber die Rolle eines Lehrers einnahmen. Da z.B. Erfahrungsberichte aus der Projektarbeit auch für Lehrpersonen interessant sein können, ist es empfehlenswert, diese Information auch in Rubrik „LehrerInnen“ zugänglich zu machen.

Wenn sich die Testperson vorwiegend an der im mittleren Bereich der Startseite angeführten Übersicht orientiert hat, wurden Informationen (bspw. Erfahrungsberichte von Schulen über Projekte oder zukünftige Informationsveranstaltungen an Universitäten) zum Teil schneller gefunden. Zwei Testerinnen nahmen diese Übersicht anfangs gar nicht wahr, sondern orientierten sich ausschließlich an den Rubriken für die Zielgruppen. Erst als sie die gewünschten Informationen über die Rubriken der Zielgruppen nicht fanden, orientierten sie sich in einem zweiten Schritt an der Übersicht auf der Startseite. Die Vielzahl an angeführten Themen, sowie die relative bunte Gestaltung - bei der die Überschriften zum Teil etwas untergehen - wurden von den Testerinnen als unübersichtlich kommentiert.

Neben den vier Zielgruppen beinhaltet die vierte Rubrik „Netzwerk“ wichtige Informationen für alle Zielgruppen von Young Science, wie etwa Informationen über kommende Veranstaltungen sowie Netzwerkkontakte bzw. Ansprechpersonen an Schulen, Universitäten und Fachhochschulen (siehe Abbildung 2). Alle Testerinnen haben erst nach einer erfolglosen Suche in den anderen Rubriken auf 
"Netzwerk" geklickt, um dort nach Kontakt- bzw. Veranstaltungsinformationen zu suchen. Aber auch wenn bei der Suche nach einer Kontaktperson an der Universität die richtige Seite angeklickt wurde, wurde bspw. eine Testerin nicht fündig, da sie den Namen der Universität in der Liste übersah. Die sichtbare Angabe der Young Science-Kontaktpersonen auf der Homepage war auch für die Workshop-TeilnehmerInnen wichtig. Einige Workshop-TeilnehmerInnen wussten jedoch nicht, dass es bereits eine Auflistung in der Rubrik „Netzwerk“ gibt.

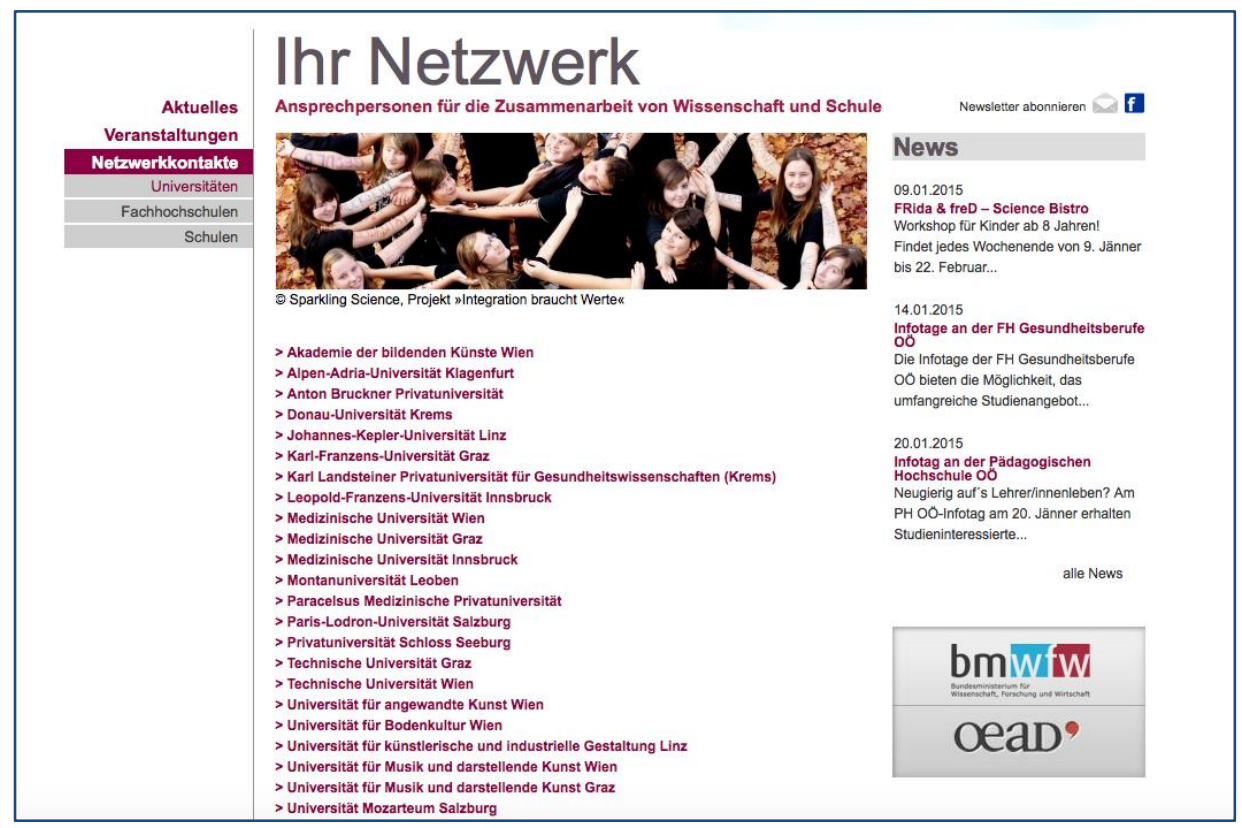

Abbildung 2: Screenshot der Liste von Young-Science Kontaktpersonen an Hochschulen (14.01.2014)

Zudem ist mit Young Science wenig vertrauten Personen nicht immer klar, dass es sich um ein Projekt mit dem Ziel der Vernetzung handelt. Dadurch bleibt unklar, was in der Rubrik „Netzwerk“ zu erwarten ist. Das zeigt sich auch darin, dass die Testerinnen diese Rubrik erst nach den anderen Rubriken angeklickt haben. „Netzwerk“ ist daher ein Begriff, der wohl die Innensicht des Zentrums gut wiederspiegelt, sich Außenstehenden aber nicht immer erschließt.

Die Suche nach Informationen auf der Themenplattform war für alle Testerinnen einfach. Die Übersichtlichkeit und der logische Aufbau wurden hervorgehoben. Bei der Suche nach Themen aus den Bereichen „Politik“ bzw. "Geschichte“ war jedoch nicht immer klar, in welcher Kategorie diese zu finden sind (siehe Abbildung 3). 


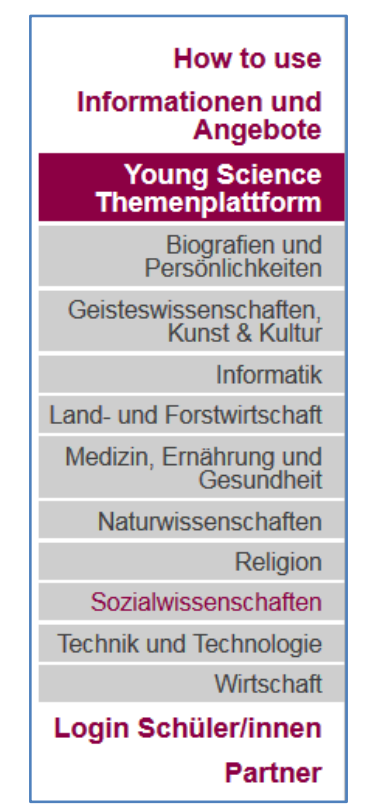

Abbildung 3: Screenshot der Gliederung der Themenplattform (14.01.2014)

Wenn Testerinnen die gesuchten Informationen nicht finden konnten, griffen zwei Testerinnen auf die Suchfunktion zurück. Auf diesem Weg wurde nach Eingabe von Schlagwörtern (wie „Kontaktperson Uni“ bei Aufgabe 1, siehe Anhang 5) die gesuchte Information sehr schnell gefunden. Andere Testerinnen wählten bei erfolgloser Suche hingegen nicht die Suchoption.

\section{Empfehlungen:}

- Gewisse Informationen (wie Erfahrungsberichte von verschiedenen AkteurInnen über die Projektarbeit) sind nur bei einzelnen Zielgruppen-Rubriken verlinkt, obwohl sie auch für andere Zielgruppen von Interesse sein können. Wie der Usability-Test gezeigt hat, suchen Webseiten-BesucherInnen vor allem in der Rubrik jener Zielgruppe, der sie sich zugehörig fühlen. Es empfiehlt sich daher stärker darauf zu achten, dass alle Information, die für eine spezifische Zielgruppe von Interesse sind, auch in der ihr zugewiesenen Rubrik zu finden sind.

- Die Auflistung der Young Science-Kontaktpersonen (derzeit in der Rubrik „Netzwerk“ zu finden) ist ein wichtiges Service und könnte auf der Webseite besser sichtbar gemacht werden, indem die Rubrik zum Beispiel in „Veranstaltungen und Kontakte“ umbenannt wird. Unter der derzeitigen Bezeichnung der Rubrik können sich Personen mit wenig oder keinem Vorwissen zu Young Science wenig vorstellen.

- Eine Auflistung der Netzwerkkontakte (siehe Abbildung 2) nach Bundesländern würde zu einer besseren Übersicht beitragen.

- Die Übersicht im mittleren Bereich der Startseite („Initiativen und Programme“) könnte die einzelnen Themenblöcke noch deutlicher hervorheben, wenn nur jeweils eine Überschrift erscheint bzw. die zweite Überschrift nur erscheint, wenn darüber gescrollt wird.

- Das Suchfeld (auf der Startseite rechts oben) könnte mithilfe einer größeren Schrift prominenter sein und so BesucherInnen der Webseite stärker auf die Möglichkeit einer Stichwortsuche aufmerksam machen. 
- Auf der Themenplattform für vorwissenschaftliche Arbeiten wurde die bestehende Gliederung in Schulfächer (auf der zweiten und dritten Ebene) nicht auf den ersten Blick erkannt. Um SchülerInnen die Suche zu erleichtern, könnte sich die Themengliederung bereits auf der ersten Ebene noch stärker an bestehenden Unterrichtsfächern orientieren. Denn es ist zu vermuten, dass SchülerInnen sich zumeist wenig unter wissenschaftlichen Disziplinen (bspw. Unterschied von Geistes- und Sozialwissenschaft) vorstellen können.

\section{Facebook}

Mit Stand Mitte Dezember 2014 hatte die Facebook-Seite von Young Science 436 Fans. Diese lebten laut ihren Angaben auf Facebook zu 81\% in Österreich, gefolgt von Deutschland mit 2\%. In Österreich ist eine klare Konzentration auf Wien zu beobachten: $43 \%$ aller Fans leben in Wien, gefolgt von Salzburg (4\%), Graz (4\%), Innsbruck (3\%) und Linz (2\%). Die Personen, die über Facebook erreicht werden, sind überwiegend weiblich (62\%) und im Alter zwischen 18 und 34 Jahren (64\%)(davon sind $31 \%$ im Alter von 18 bis 24 Jahren und 33\% im Alter von 25 bis 34 Jahren). Nur 3\% der Fans sind 17 Jahre oder jünger. 19\% der Fans sind zwischen 35 und 44 Jahre alt (siehe Grafik 6).

Grafik 6: Fans der Young Science Facebook-Seite nach Altersgruppen und Geschlecht (Quelle: Facebook)

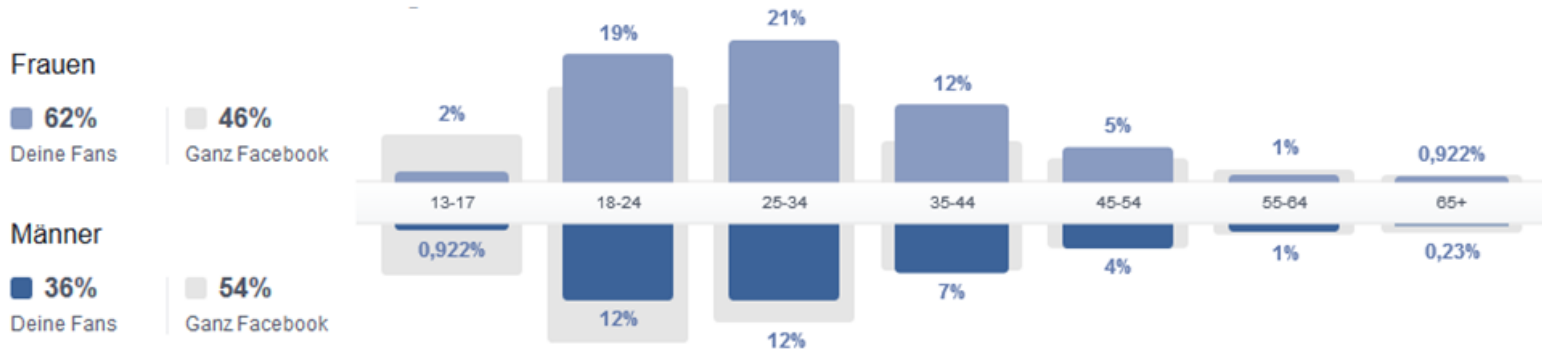

Laut einem/einer Young Science Mitarbeiterln werden über Facebook vor allem MultiplikatorInnen erreicht ${ }^{28}$ und es erweist sich als nicht einfach, sich auch mit SchülerInnen zu vernetzen. Die Altersverteilung der Personen, die Young Science auf Facebook folgen, legt jedoch nahe, dass auch einige SchülerInnen mit Young Science über Facebook vernetzt sein dürften, wenngleich es sich dem Alter nach um Personen in den Abschlussklassen handeln müsste oder um SchülerInnen, die schon vor einiger Zeit über Projekte mit Young Science in Kontakt kamen.

\section{Empfehlungen:}

- Um SchülerInnen verstärkt anzusprechen, könnten auf Facebook gezielte Aktivitäten zur Vernetzung gestartet werden. Beispielsweise könnte ein Ideen- oder Fotowettbewerb (in Zusammenhang mit einer Veranstaltung oder einem Projekt), der über Facebook veranstaltet wird, eine Möglichkeit sein, SchülerInnen verstärkt auf die Facebook-Seite aufmerksam zu machen. Auch das Posten von neuen Themen in der Themenplattform oder von Fotos und Berichten über laufende Schulprojekte könnte dazu beitragen, Schülerlnnen stärker für die Facebook-Seite von Young Science zu interessieren.

\footnotetext{
${ }^{28}$ In einem Interview verwies beispielsweise eine Young Science-Kontaktperson an einer Universität ausdrücklich darauf, den Facebook-Einträgen von Young Science zu folgen.
} 
- Twitter könnte sich als gutes Medium erweisen, um ForscherInnen stärker mit Young Science zu vernetzen. Wie eine Studie in den Vereinigten Staaten zeigt, ziehen ForscherInnen Twitter Facebook vor. ${ }^{29}$ Über Twitter könnten beispielsweise für ForscherInnen interessante Erfahrungen im Bereich Young Citizen Science kommuniziert werden.

\section{Newsletter}

Der Young Science-Newsletter wird ca. einmal im Monat versandt und kündigt Veranstaltungen, Ausschreibungen und Termine an. Dabei werden nicht nur Aktivitäten und Inhalte von Young Science selbst verschickt, sondern auch andere relevante Veranstaltungshinweise an der Schnittstelle zwischen Bildung und Wissenschaft hingewiesen. Somit werden über den Newsletter Informationen gebündelt und Synergien mit anderen Initiativen genutzt.

Mit Ende Dezember 2014 waren 683 Personen für den Young Science Newsletter angemeldet. Grafik 7 zeigt die Neuanmeldungen für den Newsletter seit 2011. Bei den Anmeldungen im Jahr 2011 dürfte es sich überwiegend um Übernahmen aus einer bestehenden Datenbank handeln. Im Jahr 2012 gab es 125 Neuanmeldungen und im Jahr 2013 konnte diese Zahl noch gesteigert werden (188 Neuanmeldungen). Allerdings ist im Jahr 2014 mit 101 Neuanmeldungen ein Einbruch zu verzeichnen. Möglicherweise hängt das damit zusammen, dass inzwischen mehr Personen den Aktivitäten von Young Science über andere Kanäle, z.B. Facebook, folgen.

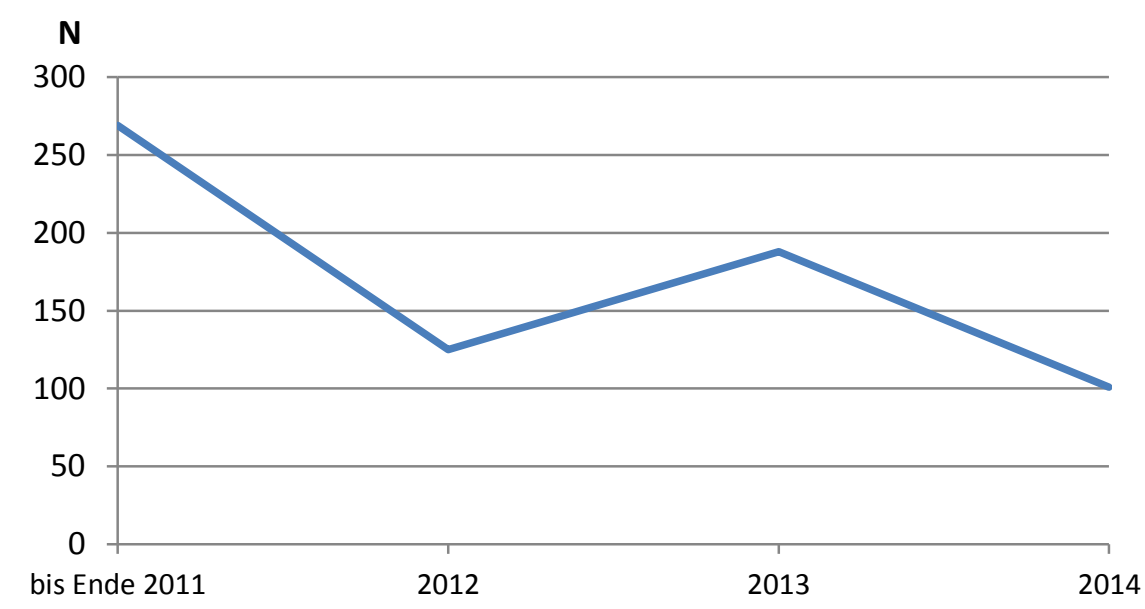

Das qualitative Feedback zum Newsletter war unterschiedlich: Je eine Person gab an, den Newsletter nie bzw. immer zu lesen. Die restlichen Befragten meinten, dass der Young Science-Newsletter gelegentlich von ihnen gelesen wird. Das Statement einer Workshop-Teilnehmerln, dass dies am Charakter von Newslettern liege, die einfach keine Priorität im Vergleich zu der Erledigung der täglichen Aufgaben haben, bringt das prägnant auf den Punkt. Eine Person betonte auch, dass Meldungen im Newsletter möglichst knapp und in wenigen Zeilen formuliert sein sollten. Trotz des gemischten qualitativen Feedbacks zum Newsletter weisen die Neuanmeldungen der letzten Jahre darauf hin, dass ein reges Interesse an diesem Informationskanal besteht.

\footnotetext{
${ }^{29}$ Yeo, Sara K. (et al.) (2014): Science Gone Social. Scientists are beginning to embrace social media as viable means of communicating with public audiences, http://www.thescientist.com/?articles.view/articleNo/40992/title/Science-Gone-Social/ [Zugriff: 15.01.2014].
} 


\section{Empfehlungen:}

- Um den Newsletter stärker zu bewerben, könnte beispielsweise im Zuge von Mails, die mit Veranstaltungsanmeldungen oder Veranstaltungsevaluierungen in Zusammenhang stehen, auf die Möglichkeit zur Newsletter-Anmeldung verwiesen werden.

- Möglichst kurz gehaltene Meldungen im Newsletter (zwei oder drei Zeilen) könnten dazu beitragen, dass der Newsletter auch bei knappen zeitlichen Ressourcen gelesen wird. Ausführliche Informationen könnten weiterhin über einen Link abrufbar sein.

\section{Young Science Projekte \& Initiativen}

\section{Gütesiegel}

Mit dem Young Science-Gütesiegel wurden 2012 erstmals Schulen ausgezeichnet, die besonders erfolgreich mit wissenschaftlichen Einrichtungen kooperierten. Die Bewerbung für die Auszeichnung stand allen Schulen und Schultypen offen.

Die Bewertungskriterien waren hoch angesetzt. Es mussten unter anderem folgende Kriterien erfüllt sein: eine regelmäßige (aktive und eigentätige) Mitarbeit der Schule an Forschungsvorhaben, forschungsorientierte Schulschwerpunkte und Unterrichtsangebote, die Unterstützung der Forschungskooperation durch die Schulorganisation, eine breite Verankerung der Forschungskooperation innerhalb der Schule sowie Qualitätssicherung der Forschungsarbeiten und langfristige Kooperationen mit Forschungseinrichtungen. Bei der Ausschreibung 2014 kam das Bewertungskriterium „geplante Vorhaben“ hinzu. Bereits 2012 ausgezeichnete Schulen konnten sich 2014 um eine Nachzertifizierung bewerben. Dafür mussten sie aber ihre Zusammenarbeit mit Forschungseinrichtungen seit 2012, die Weiterentwicklung forschungsorientierter Schulschwerpunkte und Unterrichtsangebote, die Förderung langfristiger Kooperationen sowie geplante Vorhaben nachweisen. ${ }^{30}$

Die mit dem Young Science-Gütesiegel ausgezeichneten Schulen erhalten für insgesamt zwei Jahre eine Ehrentafel, eine Urkunde, sowie das Qualitätssiegel als digitales Logo (das unter anderem für eigene Bewerbungen auf der Webseite, in Briefen, etc. verwendet werden kann). Im Jahr 2012 gab es 34 Einreichungen. ${ }^{31} 15$ Schulen wurden schließlich mit dem Young ScienceGütesiegel ausgezeichnet. Im Jahr 2014 gab es 28 Einreichungen, von denen 18 ausgezeichnet wurden. Dabei handelte es sich um sieben Nachzertifizierungen. Somit wurde das Gütesiegel seit 2012 an insgesamt 26 Schulen verliehen. ${ }^{32}$

Um das Gütesiegel zu bewerben und die Attraktivität zu erhöhen, bietet Young Science zusätzliche Anreize wie Projektmöglichkeiten für ausgezeichnete Schulen.

Bei Analyse der prämierten Schulen (der Jahre 2012 und 2014) fallen zwei Dinge auf: ein Ost/Westbzw. Stadt/Land-Gefälle, sowie die starke Homogenität der Schultypen.

\footnotetext{
${ }^{30}$ Young Science (-): Forschungspartnerschulen ausgezeichnet mit dem Young Science-Gütesiegel 2012. Young Science (-): Information zur Ausschreibung und Teilnahmebedingungen. 2. Ausschreibung 2014.

${ }^{31}$ Young Science (2012): 1. Tätigkeitsbericht. Mai 2012.

32 www.youngscience.at/guetesiegel
} 
- Ost/West-bzw. Stadt/Land-Gefälle

Werden die Auszeichnungen von 2012 und 2014 gemeinsam betrachtet, wird ein Ost-West-Gefälle ersichtlich. 23 der insgesamt 26 zertifizierten Schulen befinden sich im Burgenland, Wien, Niederösterreich, Oberösterreich und der Steiermark. In Kärnten wurden zwei Schulen ausgezeichnet und in Salzburg eine. Tirol und Vorarlberg sind die beiden Bundesländer, in denen keine Schule das Young Science- Gütesiegel erhalten hat.

Tabelle 6: Gütesiegel - Anzahl der zertifizierten Schulen nach Bundesland

\begin{tabular}{|lr|}
\hline & Anzahl der Schulen \\
\hline Kurgenland & 1 \\
\hline Ob̈rnten & 2 \\
\hline Niederösterreich & 2 \\
\hline Salzburg & 6 \\
\hline Steiermark & 1 \\
\hline Tirol & 7 \\
\hline Vorarlberg & 0 \\
\hline Wien & 0 \\
\hline Gesamt & 7 \\
\hline im Jahr 2014 sieben Nachzertifizierungen \\
\hline
\end{tabular}

Eine mögliche Ursache für die geographische Unausgewogenheit kann die geringere Anzahl an tertiären Bildungseinrichtungen und außeruniversitären Forschungseinrichtungen in westlichen Bundesländern im Vergleich zum Osten Österreichs sein. Zudem kann eine kontinuierliche und intensive Einbindung von Schulen in ländlichen Gebieten in Forschungsprojekte (wie es das Gütesiegel voraussetzt) durch längere Reisezeiten, und höhere Reisekosten (für WissenschafterInnen und SchülerInnen) sowie fehlende Infrastruktur (wie z.B. Bibliotheken) erschwert werden. Zusätzlich steht der Wettbewerb um SchülerInnen bei geringem Schulangebot (besonders in ländlichen Regionen) weitaus weniger im Vordergrund wie in Städten. Denn auch das Feedback von LehrerInnen betonte die Wettbewerbsfähigkeit der Schule beim Werben um neue SchülerInnen, die durch Schwerpunktsetzungen (wie z.B. „Forschungslabors“) und Auszeichnungen wie das Young Science Gütesiegel gestärkt wird. Dadurch wird das Gütesiegel auch gerne als „Aushängeschild“ verwendet.

- Schultypen

Werden die ausgezeichneten Schulen nach Schultyp betrachtet, zeigt sich, dass der am häufigsten ausgezeichnete Schultyp die AHS ist (13 Auszeichnungen), gefolgt von der BHS/BMS ${ }^{33}$ (10 Auszeichnungen), der Volksschule/SPZ (2 Auszeichnungen) und der Mittelschule (eine Auszeichnung). Bei den ausgezeichneten Schulen handelt es sich also fast ausschließlich um Schulen, die (u.a.) Ausbildungen anbieten, die zu einem Abschluss mit Studienberechtigung führen.

\footnotetext{
${ }^{33}$ Schulen bieten mehrere Ausbildungszweige (BHS und Fachschulen) an.
} 


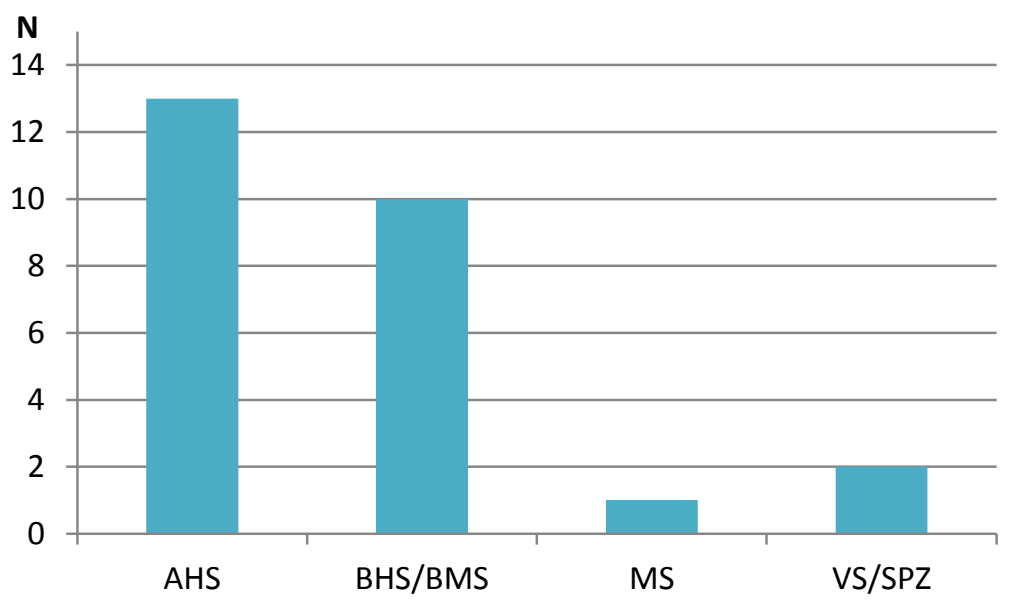

Die hoch angesetzten Bewerbungskriterien ermöglichen es vor allem Schulen, die bereits bestimmte (Forschungs-)Schwerpunkte aufweisen, Lehrpläne haben, die eine intensive Mitarbeit an Forschungsprojekten zulassen, sowie einen engagierten Lehrkörper, sich um das Gütesiegel zu bewerben. Diese relativ strengen Bewerbungskriterien sind daher für bestimmte Schultypen (wie etwa Schulen der Sekundarstufe I und Neue Mittelschule) schwer erfüllbar.

Diese hoch angesetzten Kriterien werden allerdings von den bereits ausgezeichneten Schulen als Qualitätsmerkmal angesehen, was sich im Wunsch einer Lehrperson verdeutlicht, dass das Gütesiegel durch eine mögliche Aufweichung der Bewerbungskriterien nicht zu „noch einem Gütesiegel" (Zitat Lehrer) werden soll.

Wie aus den Workshops und Interviews ersichtlich wurde, wenden sich zudem Forschungseinrichtungen auf der Suche nach Schulen bevorzugt an die Sekundarstufe II. Wie ein/e MitarbeiterIn von Young Science berichtete, werden diese Schulen einerseits aufgrund bereits gemachter Projekterfahrungen, sowie andererseits wegen der schon älteren SchülerInnen und ihren höheren Kompetenzen bevorzugt.

Das qualitative Feedback der Workshops und Interviews zum Bewerbungsprozess verdeutlicht, dass es bei der Kommunikation der Bewerbungskriterien im Rahmen der ersten Ausschreibung $2012 \mathrm{zu}$ Unklarheiten kam. Einige Schulen reagierten enttäuscht, da nicht alle wussten, dass jedes Bewertungskriterium erfüllt werden muss, um das Gütesiegel zu erhalten. Dies war, aufgrund verbesserter Kommunikation und Erfahrungswerte (von Seiten Young Science und der Schulen) bei der jüngsten Ausschreibung 2014 nicht mehr der Fall. Zudem wurde angemerkt, dass die Ausschreibung für das Gütesiegel 2014 zu einem ungünstigen Zeitpunkt (während einer Phase mit sehr vielen Schularbeiten) stattfand. Auf genauere Nachfrage konnte in der Workshop-Diskussion mit Lehrpersonen und einem Direktor jedoch auch keine Phase während des Schuljahrs gefunden werden, die alle Anwesenden passend erschien.

Laut Workshops und Interviews werden mit dem Gütesiegel ausgezeichnete Schulen sowohl intern (von Young Science) als auch extern (von Universitäten) als mögliche Partnerschulen für Projekte herangezogen. Eine Broschüre mit den ausgezeichneten Schulen wird von Young Science bei Treffen mit Hochschulen benutzt, um Schulen „ohne schlechtes Gewissen weiterzuempfehlen“ (Zitat: MitarbeiterIn Young Science). Für einige Kontaktstellen an Universitäten ist das Young ScienceGütesiegel ein wichtiges Qualitätsmerkmal, wenn Schulen als Kooperationspartner für Projekte 
gesucht werden. Andere betonen hingegen, dass sie Schulen aufgrund persönlicher Kontakte auswählen.

\section{Empfehlungen:}

- Bei der zukünftigen Schwerpunktsetzung von Young Science im Bereich Citizen Science, ist geplant, einen niederschwelligeren Zugang zu Projekten zu ermöglichen. Das hat das Potential, die Forschungs-Bildungs-Kooperation Schultypen wie Neue Mittelschulen oder Volksschulen leichter zugänglich zu machen. Dadurch könnten SchülerInnen noch stärker vor dem Übertritt in die Sekundarstufe II und damit vor der Entscheidung, ob der Weg in Richtung höhere oder berufliche Bildung eingeschlagen wird, erreicht werden. Denn wie die Erfahrungen aus einem Citizen Science Projekt zeigen, können solche Projekte gerade SchülerInnen, die ansonsten im Unterricht überwiegend passiv sind, für ein Fach motivieren. $^{34}$

- Schulen in ländlichen Regionen könnten in Zukunft gezielter angesprochen werden und bei tertiären Bildungseinrichtungen, sowie außeruniversitären Forschungseinrichtungen stärker als Projektpartnerinnen empfohlen werden.

- Speziell bei Themen wie Umwelt und Nachhaltigkeit (z.B. Pflanzen-, Tiervielfalt in Bergregionen) könnten Schulen in ländlichen Gebieten durch ihren Standort attraktive Forschungspartner darstellen. Eine erfolgreiche Forschungs-Bildungs-Kooperation im Rahmen von „Sparkling Science“ gab es etwa in der Region Stubaital in Tirol. Der Standort der Schule in einem Alpental war für das Klimaforschungsprojekt essentiell. ${ }^{35}$

Allerdings müssten bei der Projektplanung (und Einreichung) ein höherer Zeitaufwand, sowie zusätzliche Reisekosten berücksichtigt werden.

- Wie sich auch bei anderen Angeboten von Young Science gezeigt hat, ist ein persönlicher Kontakt gerade zu Beginn sehr hilfreich, um verschiedene AkteurInnen für die ForschungsBildungs-Kooperation zu gewinnen. Möglicherweise böte es sich auch hier an, dass Lehrpersonen als BotschafterInnen an anderen Schulen ihre Aktivitäten im Rahmen der Forschungs-Bildungs-Kooperation vorstellen und über das Gütesiegel informieren. Wenn das gezielt in ländlichen Regionen passiert, könnte es dazu beitragen, dem Stadt-Land- bzw. OstWest-Gefälle entgegen zu wirken.

\footnotetext{
${ }^{34}$ Manahl, Holocher-Ertl (et al.): Socientize. D5.4. Evaluation Report, http://socientize.eu/sites/default/files/socientize d5.4.pdf, S. 27.

${ }^{35} \mathrm{Mehr}$ Informationen zum Projekt unter: http://www.sparklingscience.at/de/projekte/6-top-klima-science/
} 


\section{Themenplattform für vorwissenschaftliche Arbeiten}

Das Verfassen einer vorwissenschaftlichen Abschlussarbeit (VwA) bzw. Diplomarbeit in AHS und BHS ist seit 2012 verpflichtend und wird in diesem Schuljahr (2014/15) erstmals flächendeckend in Österreich eingeführt. Die Themenplattform für vorwissenschaftliche Arbeiten ist eine OnlinePlattform auf der Young Science-Webseite, auf der aktuelle Forschungsthemen als Themenanregungen für SchülerInnen aufgelistet sind.

Die Forschungsthemen werden von WissenschafterInnen generiert und sollen Schülerlnnen und LehrerInnen die Themensuche für die vorwissenschaftlichen Arbeiten und Diplomarbeiten erleichtern. Andere Online-Plattformen (u.a. vom $\mathrm{BMBF}^{36}$ ) bieten Informationen zum Verfassen der Arbeit an, unterstützen jedoch nicht beim Finden eines geeigneten Themas. Die Themenplattform für vorwissenschaftliche Arbeiten ist seit 30. Mai 2014 online.

Über eine Auflistung möglicher Themen hinaus, können SchülerInnen direkt mit den entsprechenden WissenschafterInnen in Kontakt treten (das Angeben von persönlichen Kontaktdaten durch ForscherInnen ist optional) und Einblick in aktuelle Forschungsprojekte bekommen. Im Februar 2014 wurden insgesamt 56 Themenvorschläge auf die Plattform online gestellt. Für die weitere Themengenerierung wurden im Jahr 2014 vier Werkverträge mit Universitäten ${ }^{37}$ abgeschlossen, die je 100 Themenanregungen liefern sollten. Drei dieser Universitäten lieferten je 100 oder mehr Themenvorschläge, eine Universität übermittelte nur 63 Themenanregungen. Zusätzlich wurden insgesamt 3000 weitere Anfragen an ForscherInnen an Universitäten und außeruniversitären Forschungseinrichtungen gestellt, mit der Bitte die Themenblätter zu befüllen. ${ }^{38}$

\section{- Themengenerierung}

Zu Beginn der Themenplattform war es relativ schwierig, Themen durch (unpersönliche) Aussendungen an Universitäten und Forschungseinrichtungen zu generieren. Wie auch bei anderen Projekten und Angeboten von Young Science waren - sowohl im Rahmen der vier Werkverträge mit Universitäten als auch beim Versenden von E-Mails - persönliche Gespräche vorab hilfreich, um Fragen und Unklarheiten zu klären. Außerdem hat sich gezeigt, dass WissenschafterInnen, die bereits aufgrund von Sparkling Science oder anderen Projekten Kontakt zu Young Science hatten, vergleichsweise öfters Themenblätter befüllten. Um die in den vier Werkverträgen vereinbarten je 100 Themenvorschläge liefern zu können, war die gute Vernetzung innerhalb der eigenen Einrichtung für die durchführenden Personen von Vorteil, um KollegInnen persönlich zu Themenvorschlägen animieren zu können. Das Weiterleiten der Anfragen von einer in der Universität hierarchisch höher gestellten Person (z.B. Professorln bzw. Vizerektorln) wurde ebenso als vorteilhaft beschrieben, da sich WissenschafterInnen so verstärkt angesprochen und verpflichtet fühlten „hier auch mitzumachen" (Zitat Kontaktperson an Universität).

Zudem wurden bei der Themengenerierung einige Unklarheiten in Bezug auf das Ausfüllen der Themenblätter deutlich: Die Vorgabe, möglichst aktuelle Forschungsthemen aufzugreifen sowie zusätzlich Webseiten aktueller, relevanter Forschungsprojekte anzugeben stellten für einige WissenschafterInnen eine Hürde dar.

Zudem wurde befürchtet, durch die Angabe einer Webseite die Anonymität nicht wahren zu können. Dies war für viele ForscherInnen allerdings äußerst wichtig, da mit der Angabe persönlicher Kontaktdaten oder dem Verweis auf eine Webseite, wo diese ersichtlich sind, zusätzlich Anfragen

\footnotetext{
${ }^{36}$ Beispiele: www.literacy.at, www.ahs-vwa.at

${ }^{37}$ Universität Salzburg, Universität Graz, Universität für Bodenkultur, Montanuniversität Leoben

${ }^{38}$ Young Science (2014): „Themenplattform für vorwissenschaftliche Arbeiten und Diplomarbeiten Forschungsthemen, Links und Literaturtipps aus der Wissenschaft“, Endbericht Mai 2014.
} 
von SchülerInnen oder Betreuungspflichten befürchtet wurden, die aufgrund beschränkter zeitlicher Ressourcen nicht erwünscht waren.

\section{Empfehlungen:}

- Sollten in Zukunft erneute Werkverträge ausgegeben werden, die Personen verschiedener Organisationen mit den gleichen Aufgaben konfrontieren, empfiehlt es sich, einen Erfahrungsaustausch zwischen diesen Personen zu ermöglichen (z.B. Kontakt herstellen durch ein gemeinsames „Kick-off-meeting“). Kontaktstellen an Universitäten können von Beispielen einer erfolgreichen Themenakquisition ${ }^{39}$ profitieren. Eine Person berichtete im Interview, dass sie nur durch Zufall eine andere Person kennen lernte, die auch einen Werkvertrag zur Themenplattform hatte.

- ForscherInnen, die sich über die Themenplattform engagieren und beispielsweise SchülerInnen die Option zu einem weiteren Austausch oder persönlichen Treffen geben, könnten zukünftig gezielt über Projekt- bzw. Fördermöglichkeiten informiert werden, da diese Personen bereits Interesse an der Zusammenarbeit mit SchülerInnen zeigen. Damit hätten diese ForscherInnen auch einen konkreten Nutzen von ihrem Engagement.

- Um den Nutzen für WissenschafterInnen, sich an der Themengenerierung zu beteiligen, klarer zu kommunizieren, könnten regelmäßig Informationen zu Zugriffszahlen bzw. hochgeladenen VwAs an teilnehmende Universitäten und WissenschafterInnen geschickt werden. Auch Erfahrungsberichte über die Zusammenarbeit von ForscherInnen und SchülerInnen in Zusammenhang mit den VwAs könnten hier helfen. Generell muss aber auch hier darauf verwiesen werden, dass die Aufnahme von „Social Impact“-Indikatoren als Bewertungskriterien einer wissenschaftlichen Laufbahn sicherlich eine wichtige Voraussetzung sind, um eine größere Anzahl an ForscherInnen für die Zusammenarbeit mit SchülerInnen zu motivieren.

- Unklarheiten über die VwAs an Schulen und Universitäten In den Workshops und Interviews wurde über große Unwissenheit an den Universitäten und bei WissenschafterInnen über die VwA und deren verpflichtende Einführung an Schulen berichtet. Viele WissenschafterInnen und Kontaktstellen an Universitäten wussten über die Anforderungen einer VwA an Schulen nicht Bescheid. Die relativ neue gesetzliche Regelung, die vorwissenschaftlichen Arbeiten an AHS und BHS verpflichtend einführt, wurde von Lehrerlnnen als „große Herausforderung" bzw. „akute Baustelle“ (Zitate LehrerInnen) bezeichnet, die Unklarheiten und zusätzliche Belastungen (bspw. Themenfindung und Betreuung der Schülerlnnen) mit sich bringt. Über die VwAs herrscht also sowohl auf Seiten der Schulen als auch auf Seiten von Universitäten und WissenschafterInnen noch viel Unklarheit. Informationen und Hilfestellungen, die im letzten Jahr von mehreren Institutionen und Ministerien angeboten wurden, konnten diese nicht ausräumen. Doppelbzw. Mehrfachanfragen (von Young Science sowie von Initiativen des BMBF) an ForscherInnen und Universitäten haben teilweise für zusätzliche Verwirrung gesorgt. Diese anfänglich fehlende Absprache zwischen Young Science und anderen Initiativen hat in manchen Fällen zu Unmut geführt und sich negativ auf die Bereitschaft, Themenblätter auszufüllen bzw. andere Informationen bereitzustellen, ausgewirkt. Ein gemeinsam erstelltes Informationsblatt über die Unterschiedlichkeiten der Anfragen - einerseits, die Themengenerierung für die VwAs von Young

\footnotetext{
${ }^{39}$ Eine Person hat die Themengenerierung bspw. in eine ihrer Lehrveranstaltungen integriert. LehramtsstudentInnen haben nach aktuellen Forschungsprojekten gesucht und Themenblätter befüllt.
} 
Science, und andererseits die Suche nach KooperationspartnerInnen seitens des BMBF - wird seit kurzem bei schriftlichen Anfragen mitgeschickt.

Neben diesen Startschwierigkeiten aufgrund fehlender Absprache mit anderen Initiativen wurde auch deutlich, dass die Rolle bzw. die konkreten Aufgabenfelder von Young Science in Bezug auf die vorwissenschaftlichen Arbeiten sowohl Schulen als auch Universitäten nicht klar sein dürften. Dadurch entstehen verschiedene Erwartungen (bspw. der in Interviews geäußerte Wunsch nach Hilfestellungen für LehrerInnen im Bereich sozialwissenschaftlicher Methodik) für die Young Science nicht zuständig ist (Anm. Young Science MitarbeiterIn). Online Informationen über die VwAs, die von anderen Initiativen bereitgestellt werden, sind im Laufe des letzten Jahres auf der Webseite des Young Science Zentrums verlinkt worden, um dem allgemeinen Wunsch (besonders von Seiten der LehrerInnen) nach einer Webseite, die Informationen über die VwAs gebündelt anbietet, nachzukommen. Dadurch könnte aber auch der Eindruck entstehen, dass sich Young Science über die Themenfindung hinaus einbringt.

\section{Empfehlungen:}

- Bei der Themenplattform verdeutlichen sich Schwierigkeiten, die an Schnittstellen wie der zwischen Schulen und Wissenschaft immer wieder auftreten - durch Zuständigkeiten verschiedener Institutionen können Doppelgleisigkeiten entstehen, die bei Personen, die wenig zeitliche Ressourcen zur Verfügung haben, für Unmut sorgen. Hier scheint eine gute Abstimmung bzw. eine Kooperation der involvierten Organisationen essentiell. Eine solche Kooperation sollte in Zukunft noch stärker ausgebaut werden.

- Zudem empfiehlt sich eine klare Abgrenzung der eigenen Zuständigkeiten zu anderen Initiativen und eine verstärkte Kommunikation über Rollen und Aufgabengebiete von Young Science in Bezug auf die VwAs, damit keine Erwartungen entstehen, die nicht erfüllt werden können. Das könnte beispielsweise in der Rubrik „Informationen und Angebote“ der Themenplattform deutlich gemacht werden.

- Dennoch könnte die Themenplattform für VwAs in Zukunft weiterhin und verstärkt Links zu weiterführenden, allgemeinen Informationen über VwAs, sowie Informationen zu zuständigen Personen in den Bundesländern (z.B. Kontaktdaten der BundeslandkoordinatorInnen für VwAs) bereitstellen.

- Das Young Science Zentrum könnte in Zukunft umfassende gesetzliche Änderungen (wie die VwAs an Schulen) an Universitäten und Forschungseinrichtungen weiter kommunizieren. Dies könnte durch verstärkte Kommunikation bei diversen Veranstaltungen bzw. mithilfe eigener thematischer Newsletter geschehen.

- Themenplattform für VwAs an Schulen

Generell ist das Interesse an der Themenplattform für VwAs seitens der LehrerInnen groß und insbesondere die Vielfalt an Themen, die die Plattform bietet, wird begrüßt. Die Möglichkeit, dadurch "über das eigene Unterrichtsfach hinauszugehen“ (Zitat Lehrer) wird als Hilfestellung wahrgenommen. Durch die verpflichtende Einführung der VwAs werden LehrerInnen (als erste Ansprechpersonen für SchülerInnen) mit einer Vielzahl an unterschiedlichen Themenanfragen konfrontiert. LehrerInnen erhoffen sich, dass die Plattform (SchülerInnen und LehrerInnen) den Zugang zu fachfremden Themengebieten erleichtert. Zusätzlich wird das Zertifikat für SchülerInnen, die ihre Arbeiten auf der Themenplattform hochladen, als positiv - insbesondere für spätere Bewerbungen (auch beim Zugang zu Universitäten) - hervorgehoben. Zusätzlich können SchülerInnen die WissenschafterInnen der jeweiligen Forschungsthemen direkt kontaktieren, wenn diese einer 
Kontaktaufnahme zustimmen. Das wurde als gute Möglichkeit für SchülerInnen genannt, um Kontakte zu knüpfen und um erste Einblicke in wissenschaftliches Arbeiten zu erhalten. Das Feedback über die Gestaltung und Benutzerfreundlichkeit der Themenplattform war unterschiedlich: Einerseits wurde die Plattform als sehr übersichtlich und einfach zu navigieren beschrieben, andererseits wurden sprachliche Vereinfachungen gewünscht. Eine konkrete Anmerkung einer Landeskoordinatorin für VwAs betraf die Formulierung „Die Themenplattform dein Einstieg in die Wissenschaft" welche den Eindruck erwecke, dass SchülerInnen in Forschungsprojekten mitarbeiten. Besser wäre: „Finde ein Thema für die VwA“).

Weitere konkrete Erfahrungen mit der Plattform wird es vermutlich erst im kommenden Schuljahr geben, nachdem die ersten SchülerInnen und LehrerInnen die Plattform aktiv genutzt haben und beginnen, an ihren VwAs zu arbeiten.

\section{Empfehlung:}

- Um weitere Einblicke in die Bedienbarkeit und die Verständlichkeit des verwendeten Wortlautes der Themenplattform zu bekommen, empfiehlt sich ein Usability-Test mit SchülerInnen, die bisher nicht in das Projekt involviert waren.

- Disseminierung der Themenplattform

Bei der Disseminierung der Plattform wird - wie auch in anderen Zusammenhängen bereits öfters erwähnt - der persönliche Kontakt sehr geschätzt. LehrerInnen, WissenschafterInnen sowie Kontaktpersonen an Schulen und Universitäten schätzen es, persönlich über neue Angebote bzw. Änderungen informiert zu werden. Präsentationen der Themenplattform bei verschiedenen Veranstaltungen (z.B. Landestreffen der BildungsberaterInnen bzw. Landesschulräte) kommen sehr gut an und auch in der Zusammenarbeit mit den BundeslandkoordinatorInnen für die VwAs (die über die Plattform informiert sind und bei diversen Veranstaltungen das Angebot weiter kommunizieren) gab es bisher gute Erfahrungen.

\section{Empfehlungen:}

- Um die Bekanntheit der Themenplattform an Schulen weiter zu steigern, ist eine fortlaufende Zusammenarbeit mit regionalen Kontaktpersonen (bspw. LandesschulrätInnen, BildungsberaterInnen und BundeslandkoordinatorInnen für VwAs) essentiell. Daher empfehlen sich auch in Zukunft verstärkt persönliche Treffen und Präsentationen der Themenplattform auf bestehenden Veranstaltungen (wie etwa die Tagung der LandesschulrätInnen). Wie bisherige Erfahrungen zeigen, können so Fragen sofort geklärt und Unklarheiten ausgeräumt werden. Würden diese Fragen und Unklarheiten dokumentiert werden, könnten sie eine gute Basis für eine „Fragen \& Antworten“ Sektion oder die Anleitung auf der Themenplattform bilden.

- Die neue Kommentarfunktion auf der Themenplattform stellt eine gute Möglichkeit für Young Science-MitarbeiterInnen dar, in direkten Kontakt mit SchülerInnen zu treten und deren Fragen zeitnahe zu beantworten. Diese Blogfunktion könnte in Zukunft vermehrt an LehrerInnen bzw. SchülerInnen kommuniziert und gleichzeitig als „direkter Feedback-Kanal“ von SchülerInnen genutzt werden.

- Durch persönliche Treffen könnten auch außeruniversitären Forschungseinrichtungen stärker für das Projekt bzw. die Themengenerierung gewonnen werden. Dadurch könnte die Plattform auch gezielt in jenen Themenbereichen ausgebaut werden, die noch weniger befüllt sind. 


\section{Projekte zum Schwerpunkt Nachhaltigkeit}

Im Zuge des RIO+20 Jubiläums im Jahr 2012 wurde Young Science vom BMWF beauftragt, verschiedene Projekte für Schulen und Forschungsinstitutionen auszuschreiben.

Im Rahmen des Projekts „RIO+20“ wurden 3-wöchige Forschungspraktika im Zeitraum von Juli bis August 2012 für SchülerInnen im Alter zwischen 16 und 19 Jahren angeboten. Insgesamt waren laut Angebot 365 Praktika für jeweils 3 Wochen vorgesehen, die finanziell für SchülerInnen sowie Forschungseinrichtungen abgegolten wurden. ${ }^{40}$ Young Science erhielt 210 Praktikumsbewerbungen von denen insgesamt 114 PraktikantInnen an 49 Institutionen vermittelt wurden. ${ }^{41}$ Die Mehrheit der Institutionen war in Wien, Niederösterreich und Salzburg angesiedelt. Im Rahmen der Praktika wurden von den SchülerInnen Berichte verfasst. Die 25 besten der im Rahmen der Praktika geschriebenen Arbeiten wurden in einem Jubiläumsband publiziert und ausgezeichnet. ${ }^{42}$ Die Möglichkeit für SchülerInnen, Einblicke in die Wissenschaft zu bekommen und Kontakte zu knüpfen, sowie für WissenschafterInnen interessierte NachwuchsforscherInnen zu gewinnen, standen bei der Praktikumsinitiative im Vordergrund.

Zusätzlich zu den Praktika wurden aufgrund des nicht ausgeschöpften Budgets vier Forschungsprojekte, die sich mit den Zielen der Agenda 21 beschäftigten, an Schulen (in Salzburg, Wien, Niederösterreich und dem Burgenland) gefördert.

Aufgrund des Erfolgs von „RIO+20" gab es im darauffolgenden Jahr eine weitere Projektausschreibung zum Thema Nachhaltigkeit. Beim Projekt „Gemeinsam für Nachhaltige Entwicklung - The Future We Want" wurden Young Science-Gütesiegelschulen, die 2012 ausgezeichnet wurden, eingeladen, sich mit Projektskizzen ${ }^{43}$ zum Thema Klimawandel zu bewerben. Insgesamt haben sechs (der insgesamt 15) Gütesiegelschulen Forschungsprojekte, die sich an aktuellen Themen des Climate Change Center Austria orientierten, durchgeführt. ${ }^{44}$

Zusätzlich zu den Schulprojekten wurden im Rahmen von "Gemeinsam für eine Nachhaltige Zukunft The Future We Want" auch im Jahr 2013 insgesamt 100 Sommerpraktika in Forschungseinrichtungen für SchülerInnen angeboten. Insgesamt wurden 98 Praktika vergeben aus denen ebenso wie im Vorjahr eine Publikation mit den 25 besten Praktikumsberichten resultierte. ${ }^{45}$

\section{- Forschungspraktika}

Die Praktika wurden von SchülerInnen gut angenommen und Möglichkeiten, „einen Blick hinter die Kulissen" (Zitat MitarbeiterIn von Young Science) zu erhalten, genutzt. Die geographische Verteilung der Praktikumsplätze (vorwiegend im Osten Österreichs) machte es aber nicht möglich, allen interessierten SchülerInnen Praktika in ihrer Nähe zu vermitteln. Eine PraktikantInnen Befragung von Young Science zeigte auf, dass die Möglichkeit, die eigene Arbeit in Form einer Publikation zu veröffentlichen, zusätzlich positiv bewertet wurde. ${ }^{46}$

\footnotetext{
${ }^{40}$ BMWF (2011): Ansuchen um Einzelförderung. Beschreibung des Vorhabens (Anhang Punkt 4).

${ }^{41}$ OeAD (-): Projektdokumentation. Jubiläumsjahr RIO+20.

${ }^{42}$ Dürrstein, H. (Hrsg.) (2012): Forschung für nachhaltige Entwicklung. Wissenschafter/innen und Jugendliche ziehen Bilanz. Eine Initiative des BMWF anlässlich des Jubiläumsjahres RIO+20, StudienVerlag, Innsbruck.

${ }^{43}$ Die Forschungsvorhaben sollten aktuelle Themen des Climate Change Center Austria (CCCA) aufgreifen und eines von insgesamt 6 Themen, wie etwa Ursachen des Klimawandels bzw. Mitigation (Strategien zum Klimaschutz) behandeln.

${ }^{44}$ Young Science (-): „Gemeinsam für eine nachhaltige Entwicklung - The Future We Want“ 2. Zwischenbericht.

${ }^{45}$ Dürrstein, H., Siegele, P. (Hrsg) (2013): Gemeinsam für eine nachhaltige Entwicklung. Zukunftsvisionen von Schüler/innen und Wisenschaftler/innen. Eine Initative des BMWF 2013, StudienVerlag, Innsbruck.

${ }^{46}$ OeAD (-): Projektdokumentation. Jubiläumsjahr RIO+20.
} 
- Schulprojekte (RIO+20, „Gemeinsam für eine nachhaltige Zukunft")

Von Seiten der LehrerInnen wurden bei den Schulprojekten die Freiheit bei der Themenwahl, sowie die Aktualität des Themas „Nachhaltigkeit" sehr geschätzt. Im Workshop und in den Interviews wurde (wie schon öfters erwähnt) die persönliche und unkomplizierte Zusammenarbeit mit MitarbeiterInnen von Young Science während der Projektumsetzung positiv hervorgehoben. Bei der konkreten Umsetzung der Forschungsprojekte wurde mehrmals erwähnt, dass die persönliche Ebene und „Chemie" zwischen Schulen und den Universitäten (bzw. den zuständigen WissenschafterInnen) stimmen muss. LehrerInnen betonten insbesondere auch die positiven Aspekte einer kontinuierlichen Anwesenheit der WissenschafterInnen an der Schule. Dadurch wurde die Beziehung der SchülerInnen zu WissenschafterInnen oft sehr persönlich und einige „Berührungsängste“ abgebaut. Zusätzlich wurde die aktive und ernsthafte Einbeziehung von SchülerInnen in das Forschungsdesign und die Fragestellungen sehr geschätzt. Neben den Lernerfahrungen von SchülerInnen wurde von LehrerInnen betonten, dass sie bei diesen Projekten selbst vieles lernen und die Möglichkeit haben, ihren Wissenstand in Bezug auf aktuelle Forschungsthemen und Methodik zu erweitern.

\section{- Schwierigkeiten bei der Umsetzung von Schulprojekten}

Seitens der LehrerInnen und Kontaktstellen an Schulen wurden die geringe Flexibilität im Lehrplan, sowie Zeitmangel als größte Barrieren genannt, um neue Projekte einzureichen bzw. durchzuführen. Das Gelingen von Schulprojekten hängt stark von einem engagierten Lehrkörper, sowie interessierten Personen in der Führungsebene, die (auch bei Lehrerlnnenwechsel) Projekte mit wissenschaftlichen Einrichtungen unterstützen, ab. Zudem stellen bestimmte Strukturen an den Schulen, wie etwa Lehrpläne bzw. das Ausmaß an Freifächern, Barrieren in der Projektumsetzung dar. Zusätzlich wurde im Workshop und in den Interviews wiederholt angesprochen, dass es keine finanzielle Abgeltung für LehrerInnen gibt, die Zeit in diese Projekte investieren. Aber nicht nur von Seiten der LehrerInnen wird freiwillig Zeit investiert, sondern auch von SchülerInnen wird in manchen Projekten bestimmten Aufgaben in der Freizeit nachgegangen.

Sowohl WissenschafterInnen als auch Kontaktstellen an Universitäten betonten zwar, dass eine Zusammenarbeit mit Schulen interessant und attraktiv sei, es jedoch wenig (bis gar keine) Anerkennung für solche Projekte in der Forschungsgemeinschaft gibt. Zusätzlich ist die Verwertbarkeit der gesammelten Daten ein oft diskutiertes Thema, da ihre Qualität häufig als nicht gleichwertig mit Daten, die von WissenschafterInnen selbst gesammelt werden, erachtet wird. ${ }^{47} \mathrm{Da}$ für die wissenschaftliche Karriere Publikationen essentiell sind, können solche Zweifel an der Verwertbarkeit von Daten wissenschaftliche Publikationen erschweren.

\section{Empfehlungen:}

- Um die Zusammenarbeit mit Schulen, auch im Zuge des neuen thematischen Schwerpunktes Citizen Science in der wissenschaftlichen Gemeinschaft stärker zu bewerben, könnten Veranstaltungen für ForscherInnen hilfreich sein, bei denen WissenschafterInnen über ihre Praxiserfahrungen an Schulen berichten. Diese könnten bestimmte thematische Fokusse haben, wie bspw. „Daten sammeln mit SchülerInnen“ und somit dazu beitragen, Ängste und Zweifel bezüglich der Datenqualität auszuräumen bzw. Beispiele guter Praxis auszutauschen.

\footnotetext{
${ }^{47}$ Datenqualität ist ein Punkt, der auch in Zusammenhang mit Citizen Science Projekten viel diskutiert wird.
} 
- Ähnlich wie für WissenschafterInnen könnten thematische Austauschtreffen für LehrerInnen und Kontaktstellen an Schulen - bspw. zu Herausforderungen der Projektumsetzung - einen großen Mehrwert darstellen. Diese könnten in kleinem Rahmen (z.B. Workshop) und auch in den Bundesländern stattfinden.

- Zusätzlich zu den Austauschtreffen könnten aus den Praxiserfahrungen von LehrerInnen und WissenschafterInnen Empfehlungen verschriftlich werden und auf der Young Science Webseite online gestellt werden. Diese Empfehlungen könnten Dinge, wie etwa die Anerkennung des Engagements von Seiten aller Beteiligten (der Schulen und der Wissenschaft) beinhalten. In der konkreten Umsetzung könnte eine Anerkennung des Engagements bzw. Anreize z.B. über Zertifikate für SchülerInnen und LehrerInnen geschaffen werden. ${ }^{48}$

\section{Entwicklungspotentiale im Bereich Citizen Science}

Seit Ende 2014 betreut Young Science sieben Pilotprojekte im Bereich Citizen Science. Diese Schwerpunktsetzung von Young Science im Bereich Young Citizen Science soll in Zukunft einen niederschwelligeren Zugang zu Forschungsprojekten ermöglichen, als das derzeit beispielsweise bei Sparkling Science Projekten der Fall ist. Das dürfte die Angebote von Young Science für Schultypen wie Neue Mittelschulen oder Volksschulen attraktiver bzw. leichter realisierbar machen. Auch ForscherInnen, die laut Interviews und Workshops bei Schulprojekten derzeit eher zu höheren Schulen und SchülerInnen mit einem höheren Kompetenzniveau tendieren, könnten sich bei niederschwelligeren Projekten an andere Schultypen als AHS und BHS wenden. In einem Interview wurde zudem darauf verwiesen, dass mit kleineren Projekten auch Forscherlnnen, die bislang wenig Interesse an der Kooperation mit Schulen gezeigt haben, für die Forschungs-Bildungs-Kooperation gewonnen werden könnten. Auch der derzeitige „Boom“ des Themas Citizen Science auf europäischer Ebene, dürfte dienlich sein, um mehr ForscherInnen für die Kooperation mit Schulen zu interessieren.

Gleichzeitig bestand bei einem Interviewpartner auch die Befürchtung, dass durch die Öffnung von Programmen wie Sparkling Science die Qualität der Einbindung der SchülerInnen sinken könnte. Internationale Erfahrungen mit der Einbindung von SchülerInnen in Citizen Science Projekte sind hier hilfreich, um die Öffnung der Forschungsprojekte für neue AkteurInnen mit einer gleichzeitig qualitativ hochwertigen Einbindung von SchülerInnen zu forcieren. Wie SchülerInnen in Citizen Science Projekte eingebunden werden können und was dabei berücksichtigt werden sollte, zeigen beispielsweise die Erfahrungen aus dem Projekt Socientize. ${ }^{49}$

Young Science könnte sich in den nächsten Jahren zu einem Kompetenzzentrum im Bereich Citizen Science an Schulen weiterentwickeln, das Informationen, Erfahrungen und Beispiele guter Praxis für interessierte ForscherInnen und LehrerInnen aufbereitet und anbietet.

\footnotetext{
${ }^{48}$ siehe Holocher-Ertl, T., Kieslinger, B. (2014): Citizen Science: Definitionen, Ausprägungsformen und Aktionsmodule. Arbeitspapier BMWFW.

${ }^{49}$ Im FP7-Projekt Socientize waren SchülerInnen eng in die Umsetzung eines Experiment („Cell Spotting) eingebunden. Die Erfahrungen sind im Evaluationsbericht nachzulesen (Manahl, Holocher-Ertl (et al.): Socientize. D5.4. Evaluation Report, http://socientize.eu/sites/default/files/socientize d5.4.pdf.
} 


\section{Anhang 1: Informationen über die befragten Personen}

Personen folgender Einrichtungen wurden im Zuge dieser Studie befragt:

Workshop I (1. Dezember 2014, ZSI)

- BORG Deutsch-Wagram

- Universität für Bodenkultur Wien

- Sacre Coeur Pressbaum

- Universität Innsbruck

Worshop II (16. Dezember 2014, OeAD)

- Projekt „Young Science“

Leitfadengestützte Telefoninterviews (9. bis 18. Dezember 2014, Dauer: ca. 40-60 Minuten)

- Universität Salzburg

- Universität Graz

- Akademisches Gymnasium Graz

- Pädagogische Hochschule Salzburg

- HLFS Ursprung 


\section{Leitfaden für Workshop I}

\section{Vorstellrunde \& Einstiegsfragen}

- Wir möchten damit beginnen, dass jede/r sich kurz vorstellt und erzählt, in welchem Zusammenhang er/sie das YSZ kennen gelernt hat bzw. wie ist der Kontakt zum YSZ entstanden?

- Welche Services und Angebote des YSZ kennen Sie?

\section{Vernetzung}

Beinhaltet: Newsletter, HP, Veranstaltungen, Beratung

- Wenn wir die Services NL, HP, Veranstaltungen, Beratung unter Vernetzung subsummieren. Welches dieser Services ist für Sie das Relevanteste bzw. das Wichtigste?

- Was macht diesen Service für Sie so wichtig?

- Bei Newsletter/Webseite:

- Welche Informationen sind für Sie am relevantesten? Wozu sollte es mehr Infos geben?

- Wer kann sich an den letzten Newsletter erinnern? Was wurde damit gemacht?

- Bei Veranstaltungen:

- Welche Veranstaltungen sind für Sie die wichtigsten?

- Sind diese Veranstaltungen für Sie wegen den anderen TN oder wegen dem Thema wichtig für Sie?

- Bei Beratung:

- In welchem Zusammenhang nehmen Sie die Beratung vom YSZ in Anspruch?

- Zu welchen Themen würden Sie sich mehr Informationen wünschen?

- Was könnte an diesem Service verbessert werden?

- Welches Service hat für Sie momentan den geringsten Nutzen?

$\circ$ Woran liegt das?

○ Wie sollte so ein Service aussehen, um für Sie nützlich zu sein? Bzw. welche Infos oder Leistungen würden Sie sich erwarten?

[Nachfragen: Sie repräsentieren ja verschiedene AkteurInnen im Netzwerk (LehrerInnen, Personen an Schnittstellen in Universitäten, DirektorInnen,...). Sehen Sie das in Ihrer Rolle als ... auch so, oder sind sie anderer Meinung?]

- Die Vernetzung von SchülerInnen, LehrerInnen, FoscherInnen und Personen an der Schnittstelle zwischen Schulen und Forschung ist die zentrale Aufgabe des YSZ. Diese Personen arbeiten alle in verschiedenen Kontexten. Wie sieht das aus ihrer Rolle als ... aus, welche Barrieren oder Schwierigkeiten gibt es für Sie, sich in ein solches Netzwerk ein zu bringen?

- Gibt es eine Zielgruppe des YSZ - SchülerInnen, LehrerInnen, FoscherInnen und Personen an der Schnittstelle zwischen Schule und Forschung - mit der Sie sich mehr Austausch wünschen würden?

○ Wenn ja, wie könnte/sollte dieser Austausch aussehen? 


\section{YS-Projekte \& Initiativen}

Rio+20, The Future We Want

- Sie haben ja vorher schon einige Projekte genannt, die Ihnen im Zusammenhang mit dem Young Science Zentrum eingefallen sind, mit welchen Projekten haben Sie konkret Erfahrungen gemacht?

- Was hat Sie an diesen Projekten besonders interessiert?

- Welches Interesse gab es von Seiten Ihrer Institution mitzumachen?

- Welche Erwartungen hatten Sie an das Projekt?

- Welche Dinge sind anders verlaufen als erwartet?

- Wie hätte das Projekt aussehen müssen, um Ihren Erwartungen gerecht zu werden?

- Wenn Sie an den Verlauf des Projekts denken,

- Was hat Ihrer Meinung nach besonders gut funktioniert, und warum?

- Wo gab es Schwierigkeiten?

- Um diese Schwierigkeiten zu vermeiden, wie hätte es Ihrer Meinung nach anders gestaltet/organisiert werden können?

- Viele der Projekte (Rio +20, „The Future we want“) beinhalten ja eine enge Zusammenarbeit zwischen Schulen und WissenschafterInnen. Wie haben Sie diese Zusammenarbeit erlebt?

- Wie hat sich die Zusammenarbeit mit den Schulen bzw. WissenschafterInnen gestaltet? Welche positiven und negativen Erfahrungen haben Sie gemacht?

- Was macht Ihrer Meinung nach eine gute Zusammenarbeit zwischen Ihnen als (Schule/WissenschafterIn) und einer (Schule/Wissenschafterln) aus?

- Rückblickend auf Ihr Projekt, wo sehen Sie die Bereicherung für sich persönlich? Ihre SchülerInnen? Ihre Institution (Schule, Uni)?

\section{Themenplattform}

An LehrerIn/Direktor:

- Wird die Themenplattform an Ihrer Schule bereit genutzt?

- Wie sind Ihre ersten Eindrücke: Wie kommt das Service bei SchülerInnen und LehrerInnen an?

\section{An Personen aus Universitäten:}

- Die meisten von Ihnen waren ja in Generierung von Themen für die Themenplattform involviert: Wie waren dabei Ihre Erfahrungen?

- Brachten sich Wissenschafterlnnen gerne in dieses Projekt ein?

- Wie einfach oder schwierig war es für WissenschafterInnen, komplexe Themen für Schülerlnnen verständlich zu formulieren?

\section{Gütesiegel}

- Was ist für Sie als Lehrerin/Direktor/MitarbeiterIn einer Universität das Attraktive an einer Auszeichnung für herausragende Schulen im Bereich der Kooperation zwischen Bildungs-, und Forschungseinrichtungen, wie dem YS-Gütesiegel?

- Können Sie uns kurz von Ihren Erfahrungen mit dem YS-Gütesiegel berichten?

\section{Abschluss}

- Wo sehen Sie noch Potentiale zur Verbesserung der Zusammenarbeit zwischen Wissenschaft und Schule?

○ Welchen Beitrag könnte das YSZ dabei leisten?

- SWOT-Analyse: Jede/r soll bitte kurz für sich überlegen, worin er/sie die Stärken, Schwächen, Möglichkeiten und Barrieren des YSZ sieht, und Stichworte auf Kärtchen schreiben.

- Wenn Sie einen Wunsch ans zukünftige YSZ hätten, welcher Wunsch wäre das? 


\section{Leitfaden für Workshop II}

\section{Vernetzung}

Mapping der Netzwerkakteurlnnen auf Pinnwand.

- Welche Akteurlnnen sind „leichter" erreichbar bzw. ins Netzwerk einzubinden als andere?

○ Woran könnte das liegen?

- Gibt es Aktivitäten, die sich besonders eignen, um bestimmte Akteurlnnen anzusprechen?

- oder: Haben Sie für die „schwer erreichbaren“ AkteurInnen bestimmte Strategien entwickelt?

Nachfragen bei HP, Newsletter, Beratung, Veranstaltungen:

- Gibt es Dinge, die bei HP, Newsletter, Beratung, Veranstaltungen bei LehrerInnen, Forscherlnnen, ... besonders gut ankommen/angekommen sind?

- Gibt es Dinge, die bei Zielgruppe XXX nicht so gut funktioniert haben?

- Gibt es Überlegungen bezüglich HP, Newsletter, Beratung, Veranstaltungen für die Zukunft?

- Aus den Interviews und dem WS haben wir den Eindruck, dass die persönliche Ebene (persönlicher Kontakt v.a. per Telefon und Präsentation der Aktivitäten bei z.B. diversen Veranstaltungen) sehr wichtig ist. Wie könnte das genutzt werden, um schwer erreichbare Zielgruppen besser einzubinden?

- Wie tragen die Projekte (Themenplattform, Gütesiegel, Rio+20 \& Praktika) zur Vernetzung bei? Inwiefern können neue AkteurInnen, ins Boot' geholt werden?

Bei Personen an Schnittstellen:

- Wie sehen Sie die Rolle einer YS-Kontaktperson?

- Welche Erwartungen haben Sie an eine solche Person?

- Wie funktioniert die Etablierung von YS-Kontaktpersonen an FHs und PHs?

\section{Bei SchülerInnen:}

- Zuletzt waren SchülerInnen stärker über Veranstaltungen eingebunden. Gibt es Überlegungen, SchülerInnen stärker direkt (nicht nur indirekt über Lehrpersonen) anzusprechen?

- Zeitliche Ressourcen scheinen in Schulen und auf Universitäten ein großes Thema zu sein. Bei Veranstaltungsbesuchen ist das besonders ausschlaggebend. Gibt es Überlegungen in Richtung virtueller Meetings (z.B. auch mit Schülerlnnen)?

HP und Newsletter:

- Wird YS inzwischen selbstständig mit Infos „gefüttert“ oder muss viel selbst recherchiert werden?

\section{Beratung:}

- Welche Themen dominieren? 


\section{YS-Projekte \& Initiativen}

\section{Gütesiegel}

- Inwiefern können mit Projekten wie dem Gütesiegel auch andere Schultypen (als AHS/BHS) angesprochen werden?

- Gibt es konkrete Pläne andere Schultypen stärker mit einzubeziehen?

\section{Themenplattform}

- In den Interviews und Feedbackberichten mit Kontaktstellen an den Universitäten wurde öfters erwähnt, dass es WissenschafterInnen schwer gefallen ist, Themen für SchülerInnen verständlich aufzubereiten. Zusätzlich wurden Befürchtungen geäußert, dass die Betreuung von SchülerInnen großen Zeitaufwand bedeuten könnte.

○ Wie könnte die Themengenerierung für Wissenschafterlnnen einfacher gestaltet werden? Gibt es dazu bereits konkrete Ideen?

- Zudem würde die Betreuung und Zusammenarbeit mit SchülerInnen in der wissenschaftlichen Karriere wenig Ansehen bekommen und macht sie daher nicht sehr attraktiv.

- Wie sehen Sie Potentiale vom Young Science Zentrum hier als Sprachrohr an der Schnittstelle von Universitäten und zuständigen Ministerien zu fungieren?

- Uns ist aufgefallen, dass bestimmte Themenfelder (Wirtschaft, Geisteswissenschaften) noch nicht so befüllt sind wie andere Themenfelder - wie könnten die bisher weniger befüllten Themenbereiche noch mit Themen "gefüttert" werden?

- Weitere Pläne für die Disseminierung der Themenplattform

\section{Rio $+20 /$ The Future we want}

- Es gibt ja verschiedene Themenschwerpunkte im YSZ, wie z.B. Nachhaltigkeit in den vergangen Jahren mit Projekten wie RIO+20, "The Future we want" - das Thema "Gender" wurde auch aufgenommen und nun Citizen Science. Wie kommen diese Schwerpunkte zustande?

- Wo sehen Sie Potentiale mit diesen Schwerpunktthemen neue AkteurInnen ins Netzwerk zu holen?

- Feedback von LehrerInnen zu Schwierigkeiten in der Umsetzung von SchülerInnen Projekten: Unwissen über Methodik - Wie könnte die Rolle des YSC hier sein?

\section{Vision YS im Jahr 2020}

- Vision des YSZ im Jahr 2020

○ (neue) Stakeholder

- Themen

- Aktivitäten 


\section{Anhang 3: Interviewleitfaden}

\section{Einstiegsfragen}

- Könnten Sie mir zu Beginn erzählen, in welchem Zusammenhang Sie das YSZ kennen gelernt haben bzw. wie ist der Kontakt zum YSZ entstanden?

- Welche Services und Angebote des YSZ kennen Sie?

\section{YS-Projekte}

Schulprojekte zum Schwerpunkt Nachhaltigkeit

- Was hat Sie an diesen Projekten besonders interessiert?

- Welches Interesse gab es auch von Seiten Ihrer Institution mitzumachen?

- Welche Erwartungen hatten Sie an das Projekt?

- Welche Dinge sind anders verlaufen als erwartet?

○ Wie hätte das Projekt aussehen müssen, um Ihren Erwartungen gerecht zu werden?

- Wenn Sie an den Verlauf des Projekts denken,

- Was hat Ihrer Meinung nach besonders gut funktioniert, und warum?

- Wo gab es Schwierigkeiten?

- Um diese Schwierigkeiten zu vermeiden, wie hätte man es Ihrer Meinung nach eventuell anders gestalten/organisieren können?

- Wie hat sich die Zusammenarbeit mit den Schulen bzw. WissenschafterInnen gestaltet?

- Welche positiven und negativen Erfahrungen haben Sie gemacht?

- Was macht Ihrer Meinung nach eine gute Zusammenarbeit zwischen Ihnen als (Schule/WissenschafterIn) und einer (Schule/Wissenschafterln) aus?

- Rückblickend auf Ihr Projekt, wo sehen Sie die Bereicherung für sich persönlich? Ihre SchülerInnen? Ihre Institution (Schule, Uni)?

- Wie war der Kontakt zu YS während des Projekts?

- Haben Sie YS während des Projekts kontaktiert? (z.B. bei Fragen, Unklarheiten)

\section{An Personen an Universitäten:}

- Welches Interesse haben Sie als Wissenschafterln bei YS Projekten mitzumachen?

- Wo sehen Sie die Potentiale Ihrer Zusammenarbeit mit Schulen/SchülerInnen für Ihre wissenschaftliche Arbeit (und Karriere)?

\section{Themenplattform}

\section{An LehrerIn:}

- Wird die Themenplattform an Ihrer Schule bereit genutzt?

- Wie sind Ihre ersten Eindrücke: Wie kommt das Service bei SchülerInnen und LehrerInnen an?

\section{An Personen aus Universitäten:}

- Waren Sie in die Generierung von Themen für die Themenplattform involviert?

- Wie waren dabei Ihre Erfahrungen?

- Brachten sich Wissenschafterlnnen gerne in dieses Projekt ein?

○ Gab es Schwierigkeiten beim „Ausfüllen“?

- Wie einfach oder schwierig war es für WissenschafterInnen, komplexe Themen für SchülerInnen verständlich zu formulieren? 


\section{Gütesiegel}

An alle:

- Was ist für Sie als LehrerIn/Mitarbeiterln einer Universität das Attraktive an einer Auszeichnung für herausragende Schulen im Bereich der Kooperation zwischen Bildungs- und Forschungseinrichtungen, wie dem YS-Gütesiegel?

An Lehrerlnnen:

- Hat Ihre Schule das YS-Gütesiegel?

Wenn ja: Welche Erfahrungen haben Sie mit dem Gütesiegel gemacht?

- Welchen Nutzen hat diese Auszeichnung für eine Schule?

An MitarbeiterInnen von Universitäten:

- Welchen Nutzen hat diese Auszeichnung für Universitäten bzw. für ForscherInnen?

\section{Vernetzung}

- Wie erfahren Sie hauptsächlich von neuen Projekten, Initiativen, Förderungen an der Schnittstelle zwischen Schule und Wissenschaft?

- Wenn wir die Services des Young Science Zentrums Newsletter, Webseite, Veranstaltungen, Beratung unter Vernetzung subsummieren. Welches dieser Services ist für Sie das Relevanteste bzw. das Wichtigste?

- Was macht diesen Service für Sie so wichtig?

Newsletter

- Welche Informationen sind für Sie am relevantesten?

- Können Sie sich an den letzten Newsletter erinnern? Was haben Sie damit gemacht? (z.B. Termin eingetragen, eine Veranstaltung besucht).

- Was könnte an diesem Service verbessert werden?

Webseite

- Welche Informationen sind für Sie am relevantesten?

- Können Sie sich an Ihren letzten Besuch auf der Homepage erinnern? Welche Informationen haben Sie gesucht?

- Gibt es auf der Homepage Bereiche, die sie häufig besuchen?

- Was könnte an diesem Service verbessert werden?

Veranstaltungen

- Welche Veranstaltungen sind für Sie die wichtigsten? Bzw. gibt es eine Veranstaltung, die sie besonders positiv in Erinnerung haben?

- Wieso ist Ihnen diese Veranstaltung so positiv in Erinnerung geblieben?

- Was könnte an diesem Service verbessert werden?

\section{Beratung}

- Können Sie sich an Ihren letzten persönlichen Kontakt mit dem YSZ erinnern?

- In welchem Zusammenhang kontaktieren Sie das YSZ?

- Welches Service hat für Sie momentan den geringsten Nutzten?

$\circ$ Woran liegt das?

- Wie sollte so ein Service aussehen, um für Sie nützlich zu sein? Bzw. welche Infos oder Leistungen würden Sie sich erwarten? 
- Die Vernetzung von Personen an der Schnittstelle zwischen Schule und Forschung ist die zentrale Aufgabe des YSZ.

Akteurlnnen in diesem Netzwerk sind: LehrerInnen, BildungsberaterInnen, WissenschafterInnen, etc. Diese Personen arbeiten alle in verschiedenen Kontexten.

Wie sieht das aus ihrer Rolle als ... aus, welche Barrieren oder Schwierigkeiten gibt es für Sie, sich in ein solches Netzwerk ein zu bringen?

- Wird die Kommunikation zwischen Schulen und Universitäten zum Selbstläufer oder braucht es das YSZ weiterhin als Vermittler?

- Gibt es eine Zielgruppe des YSZ - SchülerInnen, LehrerInnen, FoscherInnen und Personen an der Schnittstelle zwischen Schule und Forschung - mit der Sie sich mehr Austausch wünschen würden?

○ Wenn ja, wie könnte/sollte dieser Austausch aussehen?

\section{Abschluss}

- Wo sehen Sie noch Potential zur Verbesserung der Zusammenarbeit zwischen Wissenschaft und Schule?

○ Welchen Beitrag könnte das YSZ dabei leisten?

- Wenn Sie einen Wunsch ans zukünftige YSZ hätten - welcher wäre das? 


\section{Anhang 4: Usability-Test der Webseite}

\section{Aufgabe 1:}

Du bist Lehrerin in der Umgebung von Wien und möchtest für deine SchülerInnen einen Besuch an der Universität Wien organisieren. Du suchst nach der richtigen Ansprechperson an der Uni dafür. Eine Kollegin hat dir empfohlen, auf www.youngscience.at nach zu sehen, an wen du dich wenden kannst.

\section{Aufgabe 2:}

Du bist Lehrer und hast von Young Science und Projekten mit Schulen und Forschungseinrichtungen gehört. Du möchtest dich in diesem Bereich möglicherweise in Zukunft engagieren und daher auf der Webseite Erfahrungsberichte von Schulen nachlesen.

\section{Aufgabe 3:}

Du bist Forscherin und überlegst eine Projekteinreichung, bei der du mit Schulen zusammenarbeiten möchtest. Du suchst nach Schulen in Ostösterreich, die sich für die Zusammenarbeit mit Forschungseinrichtungen interessieren und schon Erfahrung in diesem Bereich haben.

\section{Aufgabe 4:}

Du bist Schülerin und suchst gerade ein Thema für deine vorwissenschaftliche Arbeit. Geschichte und Politik interessieren dich besonders. Du hast von deinem Lehrer von der Themenplattform gehört. Du musst bis zur nächsten Stunden zu zwei Themen, die dich interessieren, je zwei Literaturanregungen recherchieren.

\section{Aufgabe 5:}

Du bist Bildungsberater an einer Schule in Salzburg und hast SchülerInnen, die sich für eine Ausbildung an der Fachhochschule interessieren. Du möchtest herausfinden, wann die nächsten Informationsveranstaltungen bzw. der Tag der Offenen Tür an der FH in Salzburg stattfindet. 